\title{
RADIN FORCING AND ITS ITERATIONS
}

\author{
JOHN KRUEGER
}

Abstract. We provide an exposition of supercompact Radin forcing and present several methods for iterating Radin forcing.

In this paper we give an exposition of supercompact Radin forcing using coherent sequences of ultrafilters. This version of Radin forcing includes as special cases the Prikry forcing and Magidor forcing, both the measurable and supercompact versions. We also introduce some methods for iterating Radin forcing. First we show how to iterate Radin forcing over the same cardinal infinitely many times. Secondly we show that Magidor's method of iterating Prikry forcing over different cardinals can be extended to iterate Radin forcing.

Radin forcing was introduced in [8]. Mitchell [7] presented a version of Radin forcing which uses coherent sequences of ultrafilters in place of a measure sequence. Foreman and Woodin [5] developed a supercompact version of Radin forcing using measure sequences in the context of a proof that GCH can fail for every cardinal. See [2] for a more recent exposition of Radin forcing on a measurable cardinal.

In Section 1 we review notation and prove some technical lemmas we need in the paper. Part I, consisting of Sections 2 to 7, is an exposition of supercompact Radin forcing using coherent sequences. Part II, consisting of Sections 8 and 9, presents two methods for iterating Radin forcing. Section 8 covers iterations of Radin forcing over the same cardinal. Section 9 extends Magidor's method of iterating Prikry forcing over different cardinals to Radin forcing.

\section{Notation AND BACKGROUnd}

We assume that the reader is familiar with forcing, Prikry forcing, and supercompact cardinals; see [3] or [4].

For cardinals $\kappa \leq \lambda$ with $\kappa$ regular, let $P_{\kappa} \lambda$ denote the set of $a$ in $[\lambda]^{<\kappa}$ such that $a \cap \kappa$ is an ordinal. Then $P_{\kappa} \lambda$ is a club subset of $[\lambda]^{<\kappa}$. For $a, b$ in $P_{\kappa} \lambda$, let $a \subseteq b$ if $a \subseteq b$ and $|a|<b \cap \kappa$.

In this paper, normal ultrafilter means a normal, fine, non-principal ultrafilter on some $P_{\kappa} \lambda$. By fineness we mean that for all $i<\lambda$, the set $\left\{a \in P_{\kappa} \lambda: i \in a\right\}$ is in the ultrafilter. Normality is the property that for any function $F: P_{\kappa} \lambda \rightarrow \lambda$ such that $F(a) \in a$ for all $a$, there is $\beta<\lambda$ such that the set $\{a: F(a)=\beta\}$ is in the ultrafilter. If $U$ is an ultrafilter on $P_{\kappa} \lambda$ and $A \subseteq \kappa$, we say that $U$ concentrates on $A$ if the set $\left\{a \in P_{\kappa} \lambda: a \cap \kappa \in A\right\}$ is in $U$; equivalently, $\kappa \in j(A)$ where $j: V \rightarrow \operatorname{Ult}(V, U)$.

Date: January 2007.

This work was partially supported by FWF project number P16790-N04. 
If $U_{0}$ and $U_{1}$ are normal ultrafilters on $P_{\kappa} \lambda$, let $U_{0} \triangleleft U_{1}$ if $U_{0}$ is in $\operatorname{Ult}\left(V, U_{1}\right)$ (i.e. $U_{0}$ is less than $U_{1}$ in the Mitchell ordering). This relation is transitive and well-founded.

The following lemma is straightforward.

Lemma 1.1. Suppose $U$ is a normal ultrafilter on $P_{\kappa} \lambda$ and $j: V \rightarrow \operatorname{Ult}(V, U)$. If $D$ is a subset of $P_{\kappa} \lambda$, then $j(D) \cap P_{\kappa} j$ “ $\lambda=j$ " $D$.

Suppose $\kappa \leq \lambda_{0} \leq \lambda$ and $U$ is an ultrafilter on $P_{\kappa} \lambda$. Let $U\left\lceil\lambda_{0}\right.$ be the ultrafilter on $P_{\kappa} \lambda_{0}$ defined by letting $X$ be in $U\left\lceil\lambda_{0}\right.$ iff $X \subseteq P_{\kappa} \lambda_{0}$ and $\left\{a \in P_{\kappa} \lambda: a \cap \lambda_{0} \in X\right\}$ is in $U$. If $U$ is normal and $j: V \rightarrow \operatorname{Ult}(V, U)$, then $X \in U\left\lceil\lambda_{0}\right.$ iff $j$ " $\lambda_{0} \in j(X)$.

Suppose that $\kappa$ is $\lambda$-supercompact. Then by a theorem of Solovay, $\lambda^{<\kappa}$ is equal to $\lambda^{+}$if $\operatorname{cf}(\lambda)<\kappa$ and is equal to $\lambda$ if $\operatorname{cf}(\lambda) \geq \kappa$. See [3] for a proof.

If $M$ is an inner model, we say that $M$ is $\alpha$-closed if ${ }^{\alpha} M \subseteq M$.

If $\mathbb{P}$ is a forcing poset and $p$ is in $\mathbb{P}$, let $\mathbb{P} / p$ denote the poset consisting of conditions $q \leq p$, ordered the same as in $\mathbb{P}$.

We will often use the following style of proof. Suppose $\mathbb{P}$ is a forcing poset, $G$ is generic for $\mathbb{P}$ over $V$, and $\varphi$ is a statement in the forcing language. Then $V[G] \models \varphi$ iff for all $p$ in $G$, there is $q \leq p$ such that $q$ forces $\varphi$.

A Prikry type forcing poset is a triple $\left\langle\mathbb{P}, \leq, \leq^{*}\right\rangle$ such that $\langle\mathbb{P}, \leq\rangle$ and $\left\langle\mathbb{P}, \leq^{*}\right\rangle$ are both forcing posets, $q \leq^{*} p$ implies $q \leq p$, and the triple satisfies the Prikry property: if $\varphi$ is a statement in the forcing language for $\langle\mathbb{P}, \leq\rangle$ and $p$ is in $\mathbb{P}$, then there is $q \leq^{*} p$ such that $q$ decides $\varphi$.

If $\varphi$ is a statement in the forcing language for some forcing poset, let ${ }^{0} \varphi$ denote $\varphi$ and ${ }^{1} \varphi$ denote $\neg \varphi$.

We will use the following theorem of Cummings and Shelah [1]. Suppose that $\lambda$ is a cardinal in $V$ and $\mathbb{P}$ is a $\lambda^{+}$-c.c. forcing poset. Then $\mathbb{P}$ forces that $\operatorname{cf}(\lambda)=\operatorname{cf}(|\lambda|)$.

The following lemma generalizes a well-known characterization of the Mitchell ordering on a measurable cardinal.

Lemma 1.2. Let $U_{0}$ and $U_{1}$ be normal ultrafilters on $P_{\kappa} \lambda$. For each a in $P_{\kappa} \lambda$ let $\pi_{a}: a \rightarrow$ o.t.(a) be the unique order preserving bijection. Then $U_{0} \triangleleft U_{1}$ iff there exists a function $f: P_{\kappa} \lambda \rightarrow V_{\kappa}$ such that:

(1) $\left\{a \in P_{\kappa} \lambda: f(a)\right.$ is a normal ultrafilter on $P_{(a \cap \kappa)}$ o.t. $\left.(a)\right\}$ is in $U_{1}$,

(2) For every $X \subseteq P_{\kappa} \lambda, X \in U_{0}$ iff the set of a in $P_{\kappa} \lambda$ such that

$$
X_{a}=\left\{\pi_{a}{ }^{\prime c} c: c \in X \cap P_{(a \cap \kappa)} a\right\} \in f(a)
$$

is in $U_{1}$.

Proof. Write $j: V \rightarrow M=\operatorname{Ult}\left(V, U_{1}\right)$. In $M,[a \mapsto a]=j " \lambda,[a \mapsto a \cap \kappa]=\kappa$, and $[a \mapsto$ o.t. $(a)]=\lambda$. Let $X$ be a subset of $P_{\kappa} \lambda$. We show that $j\left(a \mapsto X_{a}\right)(j " \lambda)=X$. Note that $\pi_{j "} \lambda=j^{-1}\left\lceil j\right.$ " $\lambda$ and $j(X) \cap P_{\kappa} j$ " $\lambda=j$ " $X$. So $j\left(a \mapsto X_{a}\right)(j$ " $\lambda)=$ $X_{j " \lambda}=\left\{j^{-1 " c} c: c \in j^{\prime \prime} X\right\}=X$. Therefore $\left[a \mapsto X_{a}\right]=X$ in $M$.

Suppose that $U_{0} \triangleleft U_{1}$. Let $[f]=U_{0}$ in $M$. Clearly (1) holds. For (2), $X$ is in $U_{0}$ iff $\left[a \mapsto X_{a}\right]$ is in $[f]$. On the other hand suppose (1) and (2) are true. Then (2) implies that $[f]=U_{0}$ in $M$, so $U_{0} \triangleleft U_{1}$.

Suppose that $M$ is a transitive inner model of set theory which is $\lambda^{<\kappa}$-closed. Then by Lemma 1.2, if $U_{0}$ and $U_{1}$ are normal ultrafilters on $P_{\kappa} \lambda$ which are in $M$, then $U_{0} \triangleleft U_{1}$ iff $M \mid=U_{0} \triangleleft U_{1}$.

The next lemma is a standard result about supercompact cardinals. 
Lemma 1.3. Suppose that $\kappa \leq \lambda$ are cardinals. Assume $j: V \rightarrow M$ is an elementary embedding such that $M$ is a $2^{\left(\lambda^{<\kappa}\right)}$-closed inner model, $\operatorname{crit}(j)=\kappa$, and $j(\kappa)>\lambda$. Let $U^{*}$ be the normal ultrafilter on $P_{\kappa} \lambda$ defined by letting $X$ be in $U^{*}$ if $X \subseteq P_{\kappa} \lambda$ and $j " \lambda \in j(X)$. Write $i: V \rightarrow N=\operatorname{Ult}\left(V, U^{*}\right)$. Then there is an elementary embedding $k: N \rightarrow M$ such that $j=k \circ i$ and $\operatorname{crit}(k)=\left(2^{\left(\lambda^{<\kappa}\right)}\right)^{+N}$.

Proof. Define $k$ as follows. Let $a$ be in $N$ and let $f: P_{\kappa} \lambda \rightarrow V$ be a function such that $[f]_{N}=a$. Define $k(a)=j(f)\left(j^{\prime \prime} \lambda\right)$. It is straightforward to check that $k$ is a well-defined elementary embedding and $j=k \circ i$.

If $\beta \leq \lambda$, then $\beta$ is represented by the function $a \mapsto$ o.t. $(a \cap \beta)$ in $N$. By the definition of $k, k(\beta)=$ o.t. $(j$ " $\lambda \cap j(\beta))=$ o.t. $(j$ " $\beta)=\beta$ in $M$. So $\operatorname{crit}(k)>\lambda$.

Since $N$ and $M$ are $\lambda^{<\kappa}$-closed and $\operatorname{crit}(k)>\lambda, k\left(P_{\kappa} \lambda\right)=P_{\kappa} \lambda$ and $k\left(\mathcal{P}\left(P_{\kappa} \lambda\right)\right)=$ $\mathcal{P}\left(P_{\kappa} \lambda\right)$. If $a$ is in $P_{\kappa} \lambda$, then $k(a)$ is in $k\left(P_{\kappa} \lambda\right)=P_{\kappa} \lambda$, and $\alpha \in k(a)$ iff $k(\alpha) \in k(a)$ iff $\alpha \in a$. Therefore $k \uparrow P_{\kappa} \lambda$ is the identity. By this fact and a similar argument, $k \uparrow \mathcal{P}\left(P_{\kappa} \lambda\right)$ is the identity.

We prove by induction that for all $\beta$ less than $\left(2^{\left(\lambda^{<\kappa}\right)}\right)^{+N}, k(\beta)=\beta$. Fix $\beta$ and suppose $k(\alpha)=\alpha$ for all $\alpha$ less than $\beta$. Since $\beta$ is less than $\left(2^{\left(\lambda^{<\kappa}\right)}\right)^{+N}$, there is a surjective function $s: \mathcal{P}\left(P_{\kappa} \lambda\right) \rightarrow \beta$ in $N$. By elementarity, $k(s)$ is a surjection of $k\left(\mathcal{P}\left(P_{\kappa} \lambda\right)\right)=\mathcal{P}\left(P_{\kappa} \lambda\right)$ onto $k(\beta)$. If $A$ is in $\mathcal{P}\left(P_{\kappa} \lambda\right)$, then $k(s)(A)=k(s)(k(A))=$ $k(s(A))=s(A)$, so $k(s)=s$. Therefore $k(\beta)=\beta$.

Now $\left(2^{\left(\lambda^{<\kappa}\right)}\right)^{+N}<i(\kappa)<\left(2^{\left(\lambda^{<\kappa}\right)}\right)^{+}=\left(2^{\left(\lambda^{<\kappa}\right)}\right)^{+M}$, and $k$ maps $\left(2^{\left(\lambda^{<\kappa}\right)}\right)^{+N}$ to $\left(2^{\left(\lambda^{<\kappa}\right)}\right)^{+M}$. So $\operatorname{crit}(k)=\left(2^{\left(\lambda^{<\kappa}\right)}\right)^{+N}$.

Lemma 1.4. Suppose that $\kappa \leq \lambda$ are cardinals, $M$ is a $\lambda^{<\kappa}$-closed inner model, and $U$ is a normal ultrafilter on $P_{\kappa} \lambda$ in $M$. Let $j_{U}: V \rightarrow N_{U}=\operatorname{Ult}(V, U)$ and $j_{U}^{M}: M \rightarrow M_{U}=\operatorname{Ult}(M, U)$.

(1) If $f$ is in ${ }^{\left(P_{\kappa} \lambda\right)} M$, then $[f]_{N_{U}}=[f]_{M_{U}}$. Therefore $M_{U} \subseteq N_{U}$.

(2) $j_{U} \uparrow M=j_{U}^{M}$.

Proof. We prove (1) by induction on the rank of $[f]_{M_{U}}$. Suppose that $f$ is in ${ }^{\left(P_{\kappa} \lambda\right)} M$ and (1) holds for all functions $g$ such that $[g]_{M_{U}}$ has rank less than the rank of $[f]_{M_{U}}$. By the closure of $M, f$ is in $M$ so $[f]_{M_{U}}$ is defined. We show $[f]_{M_{U}}=[f]_{N_{U}}$. Suppose that $x$ is in $[f]_{N_{U}}$. Then there is $g$ in ${ }^{\left(P_{\kappa} \lambda\right)} M$ such that $[g]_{N_{U}}=x$ and $g(a) \in f(a)$ for almost all $a$. Clearly $[g]_{M_{U}}$ is in $[f]_{M_{U}}$, and by induction $[g]_{M_{U}}=x$. The other direction is similar.

To prove (2), let $x$ be in $M$. Then $j_{U}(x)=[f]_{N_{U}}$, where $f(a)=x$ for all $a$ in $P_{\kappa} \lambda$. Since $f$ is in ${ }^{\left(P_{\kappa} \lambda\right)} M$, by (1) we have $[f]_{N_{U}}=[f]_{M_{U}}$, and clearly $[f]_{M_{U}}=j_{U}^{M}(x)$.

Suppose that $\kappa$ is a supercompact cardinal. Then there exists a function $f$ : $\kappa \rightarrow V_{\kappa}$, known as a Laver function, such that whenever $x$ is a set and $\lambda \geq \kappa$ is a cardinal such that $\lambda \geq|\operatorname{tr}(\mathrm{x})|$, there is a normal ultrafilter $U$ on $P_{\kappa} \lambda$ with elementary embedding $j: V \rightarrow \operatorname{Ult}(V, U)$ such that $j(f)(\kappa)=x$. We will also need the following variant of this fact.

Lemma 1.5. Suppose that $\kappa \leq \lambda$ are cardinals, $X$ is a subset of $\kappa$, and there is a normal ultrafilter on $P_{\kappa} 2^{2^{\left(\lambda^{<\kappa)}\right.}}$ which concentrates on $X$. Then there is a function $f: \kappa \rightarrow \kappa$ and an ultrafilter on $P_{\kappa} 2^{\left(\lambda^{<\kappa}\right)}$ which concentrates on $X$ with elementary embedding $j: V \rightarrow M$ such that $j(f)(\kappa)=\lambda$. 
Proof. Define $f: \kappa \rightarrow \kappa$ by recursion. Suppose that $f\lceil\alpha$ is defined. Assume there is a cardinal $\beta$ with $\alpha \leq \beta<\kappa$ such that for every normal ultrafilter on $P_{\alpha} 2^{\left(\beta^{<\alpha}\right)}$ concentrating on $X \cap \alpha$ with elementary embedding $j: V \rightarrow M, j(f\lceil\alpha)(\alpha)$ is not equal to $\beta$. Let $f(\alpha)$ be the least such $\beta$. Otherwise let $f(\alpha)=0$.

Suppose for a contradiction that the statement of the lemma fails for $f$. Let $\lambda_{\kappa}$ be the least cardinal such that for every normal ultrafilter on $P_{\kappa} 2^{\left(\lambda_{\kappa}^{<\kappa}\right)}$ concentrating on $X$ with elementary embedding $i: V \rightarrow M, i(f)(\kappa)$ is not equal to $\lambda_{\kappa}$. By assumption, $\lambda_{\kappa}$ exists and $\lambda_{\kappa} \leq \lambda$.

Let $U$ be a normal ultrafilter on $P_{\kappa} 2^{2^{\left(\lambda^{<\kappa}\right)}}$ concentrating on $X$ and write $j$ : $V \rightarrow M=\operatorname{Ult}(V, U)$. By the closure of $M$, the definition of $f$, and Lemma 1.4(2), $j(f)(\kappa)=\lambda_{\kappa}$. Let $U^{*}=U\left\lceil 2^{\left(\lambda^{<\kappa}\right)}\right.$ and write $i: V \rightarrow N=\operatorname{Ult}\left(V, U^{*}\right)$. Note that $U^{*}$ concentrates on $X$. Apply Lemma 1.3 to obtain $k: N \rightarrow M$ such that $j=k \circ i$ and $\operatorname{crit}(k)=\left(2^{2^{(\lambda<\kappa)}}\right)+N$. Then $k(i(f)(\kappa))=j(f)(\kappa)=\lambda_{\kappa}$, so $i(f)(\kappa)$ must be equal to $\lambda_{\kappa}$. This contradicts the definition of $\lambda_{\kappa}$.

\section{PART I: SUPERCOMPACT RADIN FORCING}

\section{Coherent Sequences of Supercompact Ultrafilters}

Let $\kappa \leq \lambda$ be cardinals. A coherent sequence of ultrafilters on $P_{\kappa} \lambda$ is a sequence

$$
\vec{U}=\left\langle U(\alpha, i): \alpha \leq \kappa, i<o^{\vec{U}}(\alpha)\right\rangle,
$$

for some partial function $o^{\vec{U}}: \kappa+1 \rightarrow O n$ satisfying the following properties. Let $g: \kappa \rightarrow V$ be the map $g(\alpha)=\left\langle U(\alpha, i): i<o^{\vec{U}}(\alpha)\right\rangle$.

(1) There is a map $\beta \mapsto \lambda_{\beta}$ defined on the domain of $o^{\vec{U}}$ such that $\lambda_{\kappa}=\lambda$, each $\lambda_{\beta} \geq \beta$ is a cardinal, and whenever $o^{\vec{U}}(\alpha)$ is defined, $\alpha$ is closed under this map. Moreover, for $\alpha$ in the domain of $o^{\vec{U}}$ and $i<o^{\vec{U}}(\alpha)$, the set $\left\{a \in P_{\alpha} \lambda_{\alpha}\right.$ : o.t. $(a)=$ $\left.\lambda_{a \cap \alpha}\right\}$ is in $U(\alpha, i)$.

(2) Each $g(\alpha)=\left\langle U(\alpha, i): i<o^{\vec{U}}(\alpha)\right\rangle$ is a sequence of normal ultrafilters on $P_{\alpha} \lambda_{\alpha}$.

(3) Each $U(\alpha, i)$ concentrates on $\alpha \cap \operatorname{dom}\left(o^{\vec{U}}\right)$, and every $\alpha$ in the domain of $o^{\vec{U}}$ is strongly inaccessible.

(4) Fix $\beta<o^{\vec{U}}(\alpha)$ and let $j_{\beta}^{\alpha}: V \rightarrow \operatorname{Ult}(V, U(\alpha, \beta))$. Then $j_{\beta}^{\alpha}(g\lceil\alpha)(\alpha)=$ $\langle U(\alpha, i): i<\beta\rangle$.

In Part II of the paper we will need to know that coherent sequences exist under certain conditions. The next two lemmas provide this information.

Proposition 2.1. Suppose that $\left\langle U_{i}: i<\beta\right\rangle$ is a sequence of normal ultrafilters on $P_{\kappa} \lambda$ for some $\beta \leq \kappa$ such that:

(1) $U_{i}\left\lceil\kappa \neq U_{j}\lceil\kappa\right.$ for $i<j<\beta$,

(2) For all $\alpha_{0}<\alpha_{1}<\beta$ there exists a function $f_{\alpha_{0}}^{\alpha_{1}}: \kappa \rightarrow V_{\kappa}$ such that, letting $j_{\alpha_{1}}: V \rightarrow \operatorname{Ult}\left(V, U_{\alpha_{1}}\right), j_{\alpha_{1}}\left(f_{\alpha_{0}}^{\alpha_{1}}\right)(\kappa)=U_{\alpha_{0}}$,

(3) There is a function $h_{0}: \kappa \rightarrow \kappa$ such that $j_{0}\left(h_{0}\right)(\kappa)=\lambda$.

Then there is a coherent sequence $\vec{U}=\left\langle U(\alpha, i): \alpha \leq \kappa, i<o^{\vec{U}}(\alpha)\right\rangle$ of ultrafilters on $P_{\kappa} \lambda$ such that $o^{\vec{U}}(\kappa)=\beta$ and $U(\kappa, i)=U_{i}$ for $i<\beta$. 
Moreover, suppose $X$ is a subset of $\kappa$ such that each $U_{i}$ concentrates on $X$. Then we can choose $\vec{U}$ so that each $U(\alpha, i)$ concentrates on $X \cap \alpha$, and the domain of $o^{\vec{U}}$ is a subset of $X$.

Proof. For $\alpha_{0}<\alpha_{1}<\beta$ define a partial function $h_{\alpha_{0}}^{\alpha_{1}}: \kappa \rightarrow \kappa$ as follows. The domain of $h_{\alpha_{0}}^{\alpha_{1}}$ is the set of $\gamma$ in $X$ such that $f_{\alpha_{0}}^{\alpha_{1}}(\gamma)$ is a normal ultrafilter on $P_{\gamma} \mu$ for some cardinal $\gamma \leq \mu<\kappa$. If $\gamma$ is such an ordinal then let $h_{\alpha_{0}}^{\alpha_{1}}(\gamma)=\mu$.

We construct a sequence $\left\langle X_{i}: i<\beta\right\rangle$ of subsets of $\kappa$ such that for $i<j<\beta$, $X_{i} \cap X_{j}=\emptyset$ and $X_{i}$ is in $U_{i}\lceil\kappa$. By (1), for distinct $i$ and $j$ below $\beta$ we can choose $X_{i}(j)$ in $\left(U_{i}\lceil\kappa) \backslash\left(U_{j}\lceil\kappa)\right.\right.$. Let $X_{i}(i)=\kappa$. Define $X_{i}^{*}=\triangle\left\{X_{i}(j): j<\beta\right\}$. Finally let $X_{i}=X_{i}^{*} \backslash \bigcup\left\{X_{j}^{*}: j<i\right\}$.

Now define a sequence $\left\langle A_{i}: i<\beta\right\rangle$ of disjoint subsets of $\kappa$ using the following recursive definition: $\alpha$ is in $A_{i}$ if

(a) $\alpha$ is in $X_{i} \cap X, \alpha>i$, and $\alpha$ is strongly inaccessible,

(b) if $\xi<\min (\{\alpha, \beta\})$, then $\alpha$ is closed under the mapping $h_{0}^{\xi}\lceil\alpha$,

(c) for all $\beta_{0}<\beta_{1}<i, h_{\beta_{0}}^{i}(\alpha)=h_{\beta_{1}}^{i}(\alpha)$, and if $i=0$ then $h_{0}(\alpha) \geq \alpha$ is a cardinal,

(d) for $\xi<i, f_{\xi}^{i}(\alpha)$ is a normal ultrafilter on $P_{\alpha} h_{\xi}^{i}(\alpha)$ which concentrates on the sets $A_{\xi} \cap \alpha$ and $X \cap \alpha$,

(e) for $\beta_{0}<\beta_{1}<i$, letting $k: V \rightarrow \operatorname{Ult}\left(V, f_{\beta_{1}}^{i}(\alpha)\right), k\left(f_{\beta_{0}}^{\beta_{1}} \uparrow \alpha\right)(\alpha)=f_{\beta_{0}}^{i}(\alpha)$.

By recursion it is straightforward to check that each $A_{i}$ is in $U_{i} \uparrow \kappa$; in particular, (e) follows from Lemma 1.4(2).

Now define $\vec{U}$ as follows. For $\alpha<\kappa$ let $o^{\vec{U}}(\alpha)=i$ iff $\alpha$ is in $A_{i}$, and let $o^{\vec{U}}(\kappa)=\beta$. For $\alpha<\kappa$ and $\xi<o^{\vec{U}}(\alpha)$, let $U(\alpha, \xi)=f_{\xi}^{o^{\vec{U}}(\alpha)}(\alpha)$, and let $U(\kappa, \xi)=U_{\xi}$. For $\alpha \leq \kappa$ let $g(\alpha)=\left\langle U(\alpha, i): i<o^{\vec{U}}(\alpha)\right\rangle$. Define $\lambda_{\alpha}=h_{0}^{o^{\vec{U}}(\alpha)}(\alpha)$ if $o^{\vec{U}}(\alpha)>0$ and let $\lambda_{\alpha}=h_{0}(\alpha)$ if $o^{\vec{U}}(\alpha)=0$.

Fix $\alpha<\kappa$ and $\xi<o^{\vec{U}}(\alpha)$. Let $k: V \rightarrow \operatorname{Ult}(V, U(\alpha, \xi))$. By (d), $\alpha$ is in $k\left(A_{\xi}\right)$, so $k\left(o^{\vec{U}}\right)(\alpha)=k(\xi)=\xi$. By the definition of $\vec{U}$ and $(\mathrm{e}), k(g\lceil\alpha)(\alpha)=$ $\left\langle k\left(f_{i}^{\xi}\right)(\alpha): i<\xi\right\rangle=\left\langle f_{i}^{o^{\vec{U}}(\alpha)}(\alpha): i<\xi\right\rangle=\langle U(\alpha, i): i<\xi\rangle$. Similarly, if $\xi<\beta$ and $j: V \rightarrow \operatorname{Ult}(V, U(\kappa, \xi))$, then $j(g)(\kappa)=\left\langle U_{i}: i<\xi\right\rangle$.

In particular, if there is a $\triangleleft$-increasing sequence $\left\langle U_{i}: i<\beta\right\rangle$ of normal ultrafilters on a cardinal $\kappa$, where $\beta \leq \kappa$, then there is a coherent sequence $\vec{U}$ on $\kappa$ with $o^{\vec{U}}(\kappa)=\beta$. We do not know whether, for example, the existence of a $\triangleleft$-increasing sequence of ultrafilters on $\kappa$ with length $\kappa^{+}$implies the existence of a coherent sequence $\vec{U}$ with $o^{\vec{U}}(\kappa)=\kappa^{+}$.

If $\vec{U}=\left\langle U(\alpha, i): \alpha \leq \kappa, i<o^{\vec{U}}(\alpha)\right\rangle$ is a coherent sequence, then let $\vec{U} \uparrow(\beta+1)$ denote the coherent sequence $\left\langle U(\alpha, i): \alpha \leq \beta, i<o^{\vec{U}}(\alpha)\right\rangle$. The next result gives a sufficient condition for a coherent sequence with the maximum possible length.

Proposition 2.2. Suppose that $\kappa \leq \lambda$ are cardinals. Assume that there is an elementary embedding $j: V \rightarrow M$, where $M$ is a $2^{\left(\lambda^{<\kappa}\right)}$-closed inner model, such that the critical point of $j$ is $\kappa, j(\kappa)>\lambda$, and there is a function $f: \kappa \rightarrow \kappa$ such that $j(f)(\kappa)=\lambda$. Then there is a coherent sequence $\vec{U}$ of ultrafilters on $P_{\kappa} \lambda$ such that $o^{\vec{U}}(\kappa)=\left(2^{\left(\lambda^{<\kappa}\right)}\right)^{+}$and $j(\vec{U}) \uparrow(\kappa+1)=\vec{U}$. 
Assume moreover that $X$ is a subset of $\kappa$ such that $\kappa$ is in $j(X)$. Then we can choose $\vec{U}=\left\langle U(\alpha, i): \alpha \leq \kappa, i<o^{\vec{U}}(\alpha)\right\rangle$ so that each $U(\alpha, i)$ concentrates on $X \cap \alpha$ and the domain of $o^{\vec{U}}$ is a subset of $X$.

Proof. Without loss of generality assume that for all $\alpha, \alpha \leq f(\alpha)$ and $f(\alpha)$ is a cardinal. Write $f(\alpha)=\lambda_{\alpha}$.

Define $g: \kappa \rightarrow V_{\kappa}$ by induction. Suppose that $\alpha<\kappa$ and $g\lceil\alpha$ is defined. If $\alpha$ is not a strongly inaccessible cardinal in $X$ closed under $f$, then $o^{\vec{U}}(\alpha)$ and $g(\alpha)$ are not defined. Otherwise let $X_{\alpha}$ denote the set of increasing sequences $\left\langle U_{i}: i<\gamma\right\rangle$ of normal ultrafilters on $P_{\alpha} \lambda_{\alpha}$, with each $U_{i}$ concentrating on $X \cap \alpha$, such that for all $\beta<\gamma$, letting $j_{\beta}: V \rightarrow \operatorname{Ult}\left(V, U_{\beta}\right), j_{\beta}\left(g\lceil\alpha)(\alpha)=\left\langle U_{i}: i<\beta\right\rangle\right.$. Note that $X_{\alpha}$ is closed under unions of chains and contains the empty set. By Zorn's Lemma, let $g(\alpha)$ be a maximal element of $X_{\alpha}$. Write $g(\alpha)=\left\langle U(\alpha, i): i\left\langle o^{\vec{U}}(\alpha)\right\rangle\right.$.

Now define $o^{\vec{U}}(\kappa)=j\left(o^{\vec{U}}\lceil\kappa)(\kappa)\right.$. Write

$$
j(g)(\kappa)=\left\langle U(\kappa, i): i<o^{\vec{U}}(\kappa)\right\rangle .
$$

Note that each $U(\kappa, i)$ concentrates on $X$. By the closure of $M$ and Lemma 1.4(2), $\vec{U}=\left\langle U(\alpha, i): \alpha \leq \kappa, i<o^{\vec{U}}(\alpha)\right\rangle$ is a coherent sequence of ultrafilters on $P_{\kappa} \lambda$.

We show that $o^{\vec{U}}(\kappa)=\left(2^{\left(\lambda^{<\kappa}\right)}\right)^{+}$. For each $i<o^{\vec{U}}(\kappa)$, any ultrafilter on $P_{\kappa} \lambda$ in $\operatorname{Ult}(V, U(\kappa, i))$ is represented by a function $P_{\kappa} \lambda \rightarrow V_{\kappa}$. So there are at most $2^{\left(\lambda^{<\kappa}\right)}$ many ultrafilters less than $U(\kappa, i)$ in the Mitchell ordering. Therefore $o^{\vec{U}}(\kappa) \leq$ $\left(2^{\left(\lambda^{<\kappa}\right)}\right)^{+}$.

Suppose for a contradiction that $o^{\vec{U}}(\kappa)<\left(2^{\left(\lambda^{<\kappa}\right)}\right)^{+}$, and we will show that $j(g)(\kappa)=\left\langle U(\kappa, i): i<o^{\vec{U}}(\kappa)\right\rangle$ is not maximal in $M$. So we will find a normal ultrafilter $U^{*}$ on $P_{\kappa} \lambda$ in $M$ concentrating on $X$ such that, letting $j^{*}: M \rightarrow \operatorname{Ult}\left(M, U^{*}\right)$, $j^{*}\left(j(g)\lceil\kappa)(\kappa)=j^{*}(g)(\kappa)=\left\langle U(\kappa, i): i<o^{\vec{U}}(\kappa)\right\rangle\right.$. Define $U^{*}$ by letting $Y$ be in $U^{*}$ iff $Y \subseteq P_{\kappa} \lambda$ and $j$ " $\lambda \in j(Y)$. Then $U^{*}$ is a normal ultrafilter on $P_{\kappa} \lambda$ which concentrates on $X$. By the closure of $M, U^{*}$ is in $M$. Let $i: V \rightarrow N=\operatorname{Ult}\left(V, U^{*}\right)$. By Lemma 1.3 there is an elementary embedding $k: N \rightarrow M$ with critical point $\left(2^{\left(\lambda^{<\kappa}\right)}\right)^{+N}$ such that $j=k \circ i$.

Write $i(g)(\kappa)=\left\langle U_{\alpha}^{*}: \alpha<\gamma\right\rangle$. Note that $k\left(U_{\alpha}^{*}\right)=U_{\alpha}^{*}$ for each $\alpha$. Now $k$ maps $i(g)(\kappa)$ to $\left\langle U(\kappa, i): i<o^{\vec{U}}(\kappa)\right\rangle$, which by assumption has length less than $\left(2^{\left(\lambda^{<\kappa}\right)}\right)^{+}$. So $i(g)(\kappa)$ has length less than $\left(2^{\left(\lambda^{<\kappa}\right)}\right)^{+N}=\operatorname{crit}(k)$. It follows that $i(g)(\kappa)$ has length $o^{\vec{U}}(\kappa)$. Therefore $i(g)(\kappa)=\left\langle U(\kappa, \alpha): \alpha<o^{\vec{U}}(\kappa)\right\rangle$. If $j^{*}: M \rightarrow \operatorname{Ult}\left(M, U^{*}\right)$, then by Lemma 1.4(2), $j^{*}(g)=i(g)$, so $j^{*}(g)(\kappa)=\left\langle U(\kappa, \alpha): \alpha<o^{\vec{U}}(\kappa)\right\rangle$.

\section{Supercompact Radin Forcing}

Let $\kappa \leq \lambda$ be cardinals and suppose that we have a coherent sequence

$$
\vec{U}=\left\langle U(\alpha, i): \alpha \leq \kappa, i<o^{\vec{U}}(\alpha)\right\rangle
$$

of ultrafilters on $P_{\kappa} \lambda$. Let $\alpha \mapsto \lambda_{\alpha}$ be the map such that each $U(\alpha, i)$ is a normal ultrafilter on $P_{\alpha} \lambda_{\alpha}$. We define a Radin forcing $\mathbb{R}(\vec{U})=\mathbb{R}$.

For each $a$ in $P_{\kappa} \lambda$ let $\pi_{a}: a \rightarrow$ o.t.(a) denote the unique order preserving bijection. Define $A_{\vec{U}}$ as the set of $a$ in $P_{\kappa} \lambda$ such that $o^{\vec{U}}(a \cap \kappa)$ is defined and

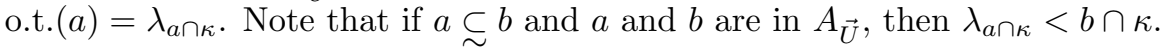


A condition in $\mathbb{R}$ is a pair

$$
\langle\vec{d}, A\rangle=\left\langle\left\langle d_{1}, \ldots, d_{n}\right\rangle, A\right\rangle
$$

such that:

(1) $A$ is in $\bigcap\left\{U(\kappa, i): i<o^{\vec{U}}(\kappa)\right\}$ and $A \subseteq A_{\vec{U}}$,

(2) $\vec{d}$ is a finite sequence, possibly empty,

(3) For each $0<i \leq n$, either $d_{i}$ is a set $a_{i}$ in $A_{\vec{U}}$ with $o^{\vec{U}}\left(a_{i} \cap \kappa\right)=0$, or $d_{i}$ is a pair $\left\langle a_{i}, A_{i}\right\rangle$ where $a_{i}$ is in $A_{\vec{U}}, o^{\vec{U}}\left(a_{i} \cap \kappa\right)>0, A_{i} \subseteq A_{\vec{U}} \cap P_{\left(a_{i} \cap \kappa\right)} a_{i}$, and the set $\left\{\pi_{a_{i}} " c: c \in A_{i}\right\}$ is in $\bigcap\left\{U\left(a_{i} \cap \kappa, \beta\right): \beta<o^{\vec{U}}\left(a_{i} \cap \kappa\right)\right\}$,

(4) For $0<i<j \leq n, a_{i} \subsetneq a_{j}$, and if $d_{j}=\left\langle a_{j}, A_{j}\right\rangle$, then for all $a$ in $A_{j}, a_{i} \sqsubseteq a$;

also, for all $a$ in $A, a_{n} \subsetneq a$.

We will abbreviate a condition $\langle\emptyset, A\rangle$ as $\langle A\rangle$ and $\left\langle\left\langle d_{1}, \ldots, d_{n}\right\rangle, A\right\rangle$ as $\left\langle d_{1}, \ldots, d_{n}, A\right\rangle$. Note that in the definition of $\mathbb{R}, n$ is the size of $\vec{d}$. We will sometimes write a condition $\langle\vec{d}, A\rangle=\left\langle d_{1}, \ldots, d_{n}, A\right\rangle$ even when $\vec{d}$ might be empty, in which case $n=0$.

Now we define the ordering on $\mathbb{R}$. Suppose that

$$
p=\left\langle d_{1}, \ldots, d_{n}, A\right\rangle
$$

and

$$
q=\left\langle e_{1}, \ldots, e_{m}, B\right\rangle
$$

are conditions in $\mathbb{R}$. Let $q \leq p$ if:

(1) $m \geq n$,

(2) $B \subseteq A$,

(3) if $n>0$, then there are $i_{1}<\ldots<i_{n} \leq m$ such that for each $0<k \leq n$,

(a) if $d_{k}$ is a set in $P_{\kappa} \lambda$, then $e_{i_{k}}=d_{k}$,

(b) if $d_{k}=\left\langle a_{k}, A_{k}\right\rangle$, then $e_{i_{k}}=\left\langle a_{k}, B_{k}\right\rangle$, where $B_{k} \subseteq A_{k}$,

(4) for each $0<l \leq m$ not equal to any $i_{k}$,

(a) if $n=0$ or if $l>i_{n}$, then either $e_{l}$ is in $A$, or $e_{l}=\left\langle b_{l}, B_{l}\right\rangle$ where $b_{l}$ is in $A$ and $B_{l}$ is a subset of $A$,

(b) if $n>0$ and $k$ is least such that $l<i_{k}$, then $d_{k}$ is of the form $\left\langle a_{k}, A_{k}\right\rangle$, and either $e_{l}$ is in $A_{k}$, or $e_{l}=\left\langle b_{l}, B_{l}\right\rangle$ where $b_{l}$ is in $A_{k}$ and $B_{l}$ is a subset of $A_{k}$. If $q \leq p$ and $n=m$, then we let $q \leq^{*} p$ and say that $q$ is a direct extension of $p$.

We will prove in Section 5 that $\left\langle\mathbb{R}, \leq, \leq^{*}\right\rangle$ is a Prikry type forcing poset.

Note that $\left\langle A_{\vec{U}}\right\rangle$ is the maximum element of $\mathbb{R}$, which we will denote by $1_{\mathbb{R}}$. If $p \leq^{*} 1_{\mathbb{R}}$ then $p=\langle B\rangle$ for some $B$.

The following lemma is immediate from the completeness of the ultrafilters.

Lemma 3.1. Let $p=\left\langle d_{1}, \ldots d_{n}, A\right\rangle$ be a condition in $\mathbb{R}$. Suppose that $\xi<\kappa$ is an ordinal such that every $d_{m}$ of the form $\left\langle a_{m}, A_{m}\right\rangle$ satisfies that $\xi<a_{m} \cap \kappa$. Then for any collection $\left\{p_{i}: i<\xi\right\}$ of direct extensions of $p$, there is $q$ such that $q \leq \leq^{*} p_{i}$ for $i<\xi$.

Similarly, if $p$ and $q$ are two conditions with the same finite sequence of elements from $P_{\kappa} \lambda$, then $p$ and $q$ are compatible. The next lemma follows from this observation.

Proposition 3.2. The poset $\mathbb{R}$ is $\left(\lambda^{<\kappa}\right)^{+}$-c.c. 
The following lemma says in effect that if $p$ is a condition in which $A$ appears as a measure one set, then there are many ways to extend $p$ to include elements from A.

Lemma 3.3. Suppose that $A$ is in $\bigcap\left\{U(\kappa, i): i<o^{\vec{U}}(\kappa)\right\}$. Let $X$ be the set of a in $A$ such that the set

$$
\left\{\pi_{a} " c: c \in A \cap P_{(a \cap \kappa)} a\right\}
$$

is in $\bigcap\left\{U(a \cap \kappa, i): i<o^{\vec{U}}(a \cap \kappa)\right\}$. Then $X$ is in $\bigcap\left\{U(\kappa, i): i<o^{\vec{U}}(\kappa)\right\}$

Suppose that $\langle b, B\rangle$ appears in $p$ for some $p$ in $\mathbb{R}$. Let $Y$ be the set of a in $P_{(b \cap \kappa)} b$ such that the set $\left\{\pi_{a} " c: c \in B \cap P_{(a \cap \kappa)} a\right\}$ is in $\bigcap\left\{U(a \cap \kappa, i): i<o^{\vec{U}}(a \cap \kappa)\right\}$. Then the set $\left\{\pi_{b} " c: c \in Y\right\}$ is in $\bigcap\left\{U(b \cap \kappa, i): i<o^{\vec{U}}(b \cap \kappa)\right\}$.

Proof. Suppose that $\beta<o^{\vec{U}}(\kappa)$ and write $j_{\beta}: V \rightarrow \operatorname{Ult}(V, U(\kappa, \beta))$. We prove that $j_{\beta}$ " $\lambda \in j_{\beta}(X)$. Note that $\pi_{j_{\beta} \text { " } \lambda}=j_{\beta}^{-1}\left\lceil j_{\beta}\right.$ " $\lambda$. By Lemma 1.1, $j_{\beta}(A) \cap P_{\kappa}\left(j_{\beta}\right.$ " $\left.\lambda\right)=$ $j_{\beta}$ " $A$, and for all $j_{\beta}(b)$ in this set, $\left(\pi_{j^{\prime \prime} \lambda}\right) " j_{\beta}(b)=j_{\beta}^{-1} "\left(j_{\beta} " b\right)=b$. Therefore

$$
\left\{\pi_{\left(j_{\beta} " \lambda\right)} " c: c \in j_{\beta}(A) \cap P_{\kappa}\left(j_{\beta} " \lambda\right)\right\}=A,
$$

which is in $\bigcap\{U(\kappa, i): i<\beta\}=\bigcap\left\{U\left(j_{\beta} " \lambda \cap j_{\beta}(\kappa), i\right): i<o^{\vec{U}}\left(j_{\beta} " \lambda \cap j_{\beta}(\kappa)\right)\right\}$. So $j_{\beta}$ " $\lambda$ is in $j_{\beta}(X)$.

Fix $\langle b, B\rangle$ as in the second statement. Let $\beta<o^{\vec{U}}(b \cap \kappa)$ and write $j_{\beta}: V \rightarrow$ $\operatorname{Ult}(V, U(b \cap \kappa, \beta))$. We show that $j_{\beta} "\left(\lambda_{(b \cap \kappa)}\right)$ is in the set $j_{\beta}\left(\left\{\pi_{b} " c: c \in Y\right\}\right)$. Let $d=j_{\beta}$ "b. We claim that $j_{\beta}\left(\pi_{b}\right) " d=j_{\beta}$ " $\left(\lambda_{(b \cap \kappa)}\right)$. For $\xi$ is in $j_{\beta}\left(\pi_{b}\right)$ " $d$ iff there is $\gamma$ in $b$ such that $\xi=j_{\beta}\left(\pi_{b}\right)\left(j_{\beta}(\gamma)\right)=j_{\beta}\left(\pi_{b}(\gamma)\right)$ iff $\xi$ is in $j_{\beta}$ " $\left(\lambda_{(b \cap \kappa)}\right)$. So it suffices to show that $d$ is in $j_{\beta}(Y)$. Note that $\pi_{d}=\left(\pi_{b} \circ j_{\beta}^{-1}\right) \uparrow j_{\beta}$ " $b$ and $j_{\beta}(B) \cap P_{\left(d \cap j_{\beta}(\kappa)\right)} d=j_{\beta}$ "B. So

$$
\left\{\pi_{d} " c: c \in j_{\beta}(B) \cap P_{\left(d \cap j_{\beta}(\kappa)\right)} d\right\}=\left\{\left(\pi_{b} \circ j_{\beta}^{-1}\right) " c: c \in j_{\beta} " B\right\}=\left\{\pi_{b} " e: e \in B\right\},
$$

which is in $\bigcap\{U(b \cap \kappa, i): i<\beta\}=\bigcap\left\{U\left(d \cap j_{\beta}(\kappa), i\right): i<j_{\beta}(\vec{U})\left(d \cap j_{\beta}(\kappa)\right)\right\}$.

\section{FACtorization}

Let $p=\left\langle d_{1}, \ldots, d_{n}, A\right\rangle$ be a condition in $\mathbb{R}$. Fix $0<m \leq n$ and suppose $d_{m}=\left\langle a_{m}, A_{m}\right\rangle$ (so in particular, $o^{\vec{U}}\left(a_{m} \cap \kappa\right)>0$ ). Define $p^{>m}=\left\langle d_{m+1}, \ldots, d_{n}, A\right\rangle$. Clearly $p^{>m}$ is in $\mathbb{R}$. Define $p^{\leq m}=\left\langle e_{1}, \ldots, e_{m-1}, B\right\rangle$ as follows. Let $B=\left\{\pi_{a_{m}}\right.$ "c : $\left.c \in A_{m}\right\}$, which is in $\bigcap\left\{U\left(a_{m} \cap \kappa, i\right): i<o^{\vec{U}}\left(a_{m} \cap \kappa\right)\right\}$. Fix $0<i<m$. If $d_{i}$ is in $P_{\kappa} \lambda$, then $o^{\vec{U}}\left(d_{i} \cap \kappa\right)=0$ and $d_{i}$ is in $P_{\left(a_{m} \cap \kappa\right)} a_{m}$. In this case let $e_{i}=\pi_{a_{m}}$ " $d_{i}$. Otherwise $d_{i}=\left\langle a_{i}, A_{i}\right\rangle$. Then $a_{i}$ is in $P_{\left(a_{m} \cap \kappa\right)} a_{m}$. Let $e_{i}=\left\langle b_{i}, B_{i}\right\rangle$, where $b_{i}=\pi_{a_{m}}$ " $a_{i}$ and $B_{i}=\left\{\pi_{a_{m}}\right.$ "c $\left.c: c \in A_{i}\right\}$. The reader can check that $p^{\leq m}$ is a condition in the Radin forcing $\mathbb{R}\left(\vec{U} \uparrow\left(\left(a_{m} \cap \kappa\right)+1\right)\right)$. We will abbreviate $\mathbb{R}\left(\vec{U} \uparrow\left(\left(a_{m} \cap \kappa\right)+1\right)\right) / p^{\leq m}$ as $\mathbb{R} / p \leq m$.

Lemma 4.1 (Factorization Lemma I). Suppose that $p=\left\langle d_{1}, \ldots, d_{n}, A\right\rangle$ is a condition in $\mathbb{R}, 0<m \leq n$, and $d_{m}=\left\langle a_{m}, A_{m}\right\rangle$. Then there is

$$
i: \mathbb{R} / p \rightarrow \mathbb{R} / p^{\leq m} \times \mathbb{R} / p^{>m} \text {. }
$$

which is an isomorphism with respect to both $\leq$ and $\leq^{*}$. 
Suppose that $q=\left\langle e_{1}, \ldots, e_{m}, B\right\rangle$ is a condition in $\mathbb{R}$ such that for all $0<i \leq m$, $e_{i}$ is in $P_{\kappa} \lambda$. Then there is

$$
j: \mathbb{R} / q \rightarrow \mathbb{R} /\langle B\rangle
$$

which is an isomorphism with respect to both $\leq$ and $\leq^{*}$.

Proof. Define $i$ as follows. Let $u=\left\langle f_{1}, \ldots, f_{l}, C\right\rangle$ be a condition in $\mathbb{R}$ below $p$. Fix $j \leq l$ with $f_{j}=\left\langle a_{m}, C_{j}\right\rangle$. Define $i(u)=\left\langle u^{\leq j}, u^{>j}\right\rangle$.

Now we define $j$. Suppose that $s=\left\langle g_{1}, \ldots, g_{k}, D\right\rangle$ is below $q$. Then the sequence $g_{1}, \ldots, g_{m}$ is exactly $e_{1}, \ldots, e_{m}$. Let $j(s)=\left\langle g_{m+1}, \ldots g_{k}, D\right\rangle$.

\section{The Prikry Property}

Now we turn to proving that $\mathbb{R}$ satisfies the Prikry property. First we show that it suffices to consider only direct extensions of $1_{\mathbb{R}}$.

Lemma 5.1. Suppose that for any coherent sequence $\vec{U}$, if $\varphi$ is a statement in the forcing language for $\mathbb{R}(\vec{U})$ and $p \leq^{*} 1_{\mathbb{R}(\vec{U})}$, then there exists $q \leq^{*} p$ which decides $\varphi$. Then for any coherent sequence $\vec{U}, \mathbb{R}(\vec{U})$ satisfies the Prikry property.

Proof. We prove the lemma by induction. Suppose that for all $\mu<\kappa$, if $\vec{U}$ is a coherent sequence on $P_{\mu} \lambda$ for some $\lambda$, then $\mathbb{R}(\vec{U})$ satisfies the Prikry property. Let $\vec{U}$ be a coherent sequence on $P_{\kappa} \lambda$ for some $\lambda$ and let $\mathbb{R}=\mathbb{R}(\vec{U})$. Consider a condition $p=\left\langle d_{1}, \ldots, d_{n}, A\right\rangle$ in $\mathbb{R}$ with $n>0$.

Suppose first that each $d_{i}$ is in $P_{\kappa} \lambda$. Then by Lemma 4.1 there is an isomorphism $j: \mathbb{R} / p \cong \mathbb{R} /\langle A\rangle$. Note that $j(p)=\langle A\rangle$, which is a direct extension of $1_{\mathbb{R}}$. By assumption $\langle A\rangle$ can be directly extended to decide any particular forcing statement, so $p$ can as well.

Otherwise fix $m \leq n$ maximal such that $d_{m}=\left\langle a_{m}, A_{m}\right\rangle$. Combining the maps $i$ and $j$ in Lemma 4.1, there is an isomorphism $k: \mathbb{R} / p \cong \mathbb{R} / p^{\leq m} \times \mathbb{R} /\langle A\rangle$. Let $\varphi$ be a statement in the forcing language for $\mathbb{R}$.

Sublemma 5.2. There is a dense set $D$ of $r$ in $\mathbb{R} / p^{\leq m}$ for which there exists $t \leq^{*}\langle A\rangle$ such that $\langle r, t\rangle$ decides $\varphi$.

Proof. Let $q$ in $\mathbb{R} / p^{\leq m}$ be given. Reverse the order of the product to $\mathbb{R} /\langle A\rangle \times$ $\mathbb{R} / p^{\leq m}$. Let $\dot{s}$ be an $\mathbb{R} /\langle A\rangle$-name for a condition below $q$ which decides $\varphi$ over the generic extension by $\mathbb{R} /\langle A\rangle$. For each $r \leq q$ let $\langle A(r)\rangle$ be a direct extension of $\langle A\rangle$ which decides whether $\dot{s}=r$. Since $\left|\mathbb{R} / p^{\leq m}\right|\langle\kappa, t=\langle\bigcap\{A(r): r \leq q\}\rangle$ is a condition. Clearly there must exist $r \leq q$ such that $t$ forces $\dot{s}=r$. Fix $t^{\prime} \leq * t$ which decides for some $l<2$ that $r$ forces ${ }^{l} \varphi$. Then $\left\langle t^{\prime}, r\right\rangle$ forces ${ }^{l} \varphi$, and therefore $\left\langle r, t^{\prime}\right\rangle$ forces ${ }^{l} \varphi$.

For each $r$ in the dense set $D$ described in the sublemma, choose $\langle B(r)\rangle$ directly below $\langle A\rangle$ such that $\langle r,\langle B(r)\rangle\rangle$ decides $\varphi$. Let $B=\bigcap\{B(r): r \in D\}$. Then $\mathbb{R} / p^{\leq m}$ forces that $B$ decides $\varphi$. By induction the poset $\mathbb{R} / p^{\leq m}$ satisfies the Prikry property. So find $q \leq^{*} p^{\leq m}$ which decides which way that $B$ decides $\varphi$. Then $\langle q,\langle B\rangle\rangle$ decides $\varphi$. It follows that $k^{-1}(\langle q,\langle B\rangle\rangle)$ is a direct extension of $p$ which decides $\varphi$.

We will need the next two lemmas to prove the Prikry property. For the remainder of this section let $I$ denote the set of sequences $\vec{d}=\left\langle d_{1}, \ldots, d_{n}\right\rangle$ such that for some $B,\left\langle d_{1}, \ldots, d_{n}, B\right\rangle$ is in $\mathbb{R}$. 
Lemma 5.3. Suppose that $U$ is a normal ultrafilter on $P_{\kappa} \lambda$. Let $F: P_{\kappa} \lambda \rightarrow I$ be a function such that, letting $a_{1}, \ldots, a_{n}$ denote the finite sequence from $P_{\kappa} \lambda$ appearing in $F(a), a_{n} \subseteq a$. Then there is $X$ in $U$ such that $F$ is constant on $X$.

Proof. Write $j: V \rightarrow M=\operatorname{Ult}(V, U)$ and let $j(F)(j “ \lambda)=\left\langle d_{1}, \ldots, d_{n}\right\rangle$. For $0<m \leq n$ write $d_{m}=a_{m}$ or $d_{m}=\left\langle a_{m}, A_{m}\right\rangle$. Then each $a_{m}$ is in $P_{\kappa} j$ " $\lambda$ and each $A_{m}$ is a subset of $P_{\kappa} j^{\text {" }} \lambda$ with size less than $\kappa$. Let $b_{m}=j^{-1}$ " $a_{m}$, and if $A_{m}$ is defined let $B_{m}=\left\{j^{-1 " b}: b \in A_{m}\right\}$. Define $\left\langle e_{1}, \ldots, e_{n}\right\rangle$ by letting $e_{m}=\left\langle b_{m}, B_{m}\right\rangle$ if $B_{m}$ is defined, otherwise $e_{m}=b_{m}$. Then $j\left(\left\langle e_{1}, \ldots, e_{n}\right\rangle\right)=\left\langle d_{1}, \ldots, d_{n}\right\rangle=j(F)(j$ " $\lambda)$, and by elementarity $\left\langle e_{1}, \ldots, e_{n}\right\rangle$ is in $I$. Let $X$ be the set of $a$ such that $F(a)=$ $\left\langle e_{1}, \ldots, e_{n}\right\rangle$.

Lemma 5.4. Suppose that $U$ is a normal ultrafilter on $P_{\kappa} \lambda$. Let $\langle X(\vec{d}): \vec{d} \in I\rangle$ be a sequence such that each $X(\vec{d})$ is in $U$. Define the diagonal intersection $\triangle\{X(\vec{d})$ : $\vec{d} \in I\}$ by letting a be in $\triangle\{X(\vec{d}): \vec{d} \in I\}$ iff $a$ is in $X(\vec{d})$ for any $\vec{d}$ in $I$ such that every $b$ in $P_{\kappa} \lambda$ appearing in $\vec{d}$ satisfies that $b \subseteq a$. Then $\triangle\{X(\vec{d}): d \in I\}$ is in $U$.

Proof. Suppose for a contradiction that the diagonal intersection is not in $U$, and let $Y$ be its complement. Define $F: Y \rightarrow I$ by letting $F(a)$ be some member of $I$ below $a$ such that $a$ is not in $X(F(a))$. By Lemma 5.3 there is a set $X \subseteq Y$ in $U$ and $\vec{d}$ in $I$ such that $F(a)=\vec{d}$ for all $a$ in $X$. But then $X \cap X(\vec{d})$ is empty, which is impossible.

Proposition 5.5. The poset $\mathbb{R}$ satisfies the Prikry Property.

Proof. Let $p=\langle A\rangle$ be a condition in $\mathbb{R}$ which is a direct extension of $1_{\mathbb{R}}$ and let $\varphi$ be a statement in the forcing language. We prove that there is a direct extension of $p$ which decides $\varphi$. The proof will follow closely the same argument from [2].

Associate to each $\vec{d}$ in $I$ and $a$ in $A$ an integer $l(\vec{d}, a)<2$ if there exists a condition below $\langle A\rangle$ which forces ${ }^{l(\vec{d}, a)} \varphi$ whose finite sequence is either $\vec{d}^{\wedge}\langle a, C\rangle$ for some $C$, or $\vec{d}^{\wedge} a$ if $o^{\vec{U}}(a \cap \kappa)=0$. Otherwise let $l(\vec{d}, a)=2$. For each $\vec{d}$ in $I$ and $i<o^{\vec{U}}(\kappa)$, let $A(\vec{d}, i) \subseteq A$ be a set in $U(\kappa, i)$ such that for all $a$ and $b$ in $A(\vec{d}, i), l(\vec{d}, a)=l(\vec{d}, b)$. Define $A(i)^{*}=\triangle\{A(\vec{d}, i): \vec{d} \in I\}$, which is in $U(\kappa, i)$ by Lemma 5.4. Define $A(0)=A(0)^{*} \cap\left\{a \in P_{\kappa} \lambda: o^{\vec{U}}(a \cap \kappa)=0\right\}$. For $i>0$ let $A(i)=A(i)^{*} \cap\left\{a \in P_{\kappa} \lambda: o^{\vec{U}}(a \cap \kappa)>0\right\}$. Finally, let $B=\bigcup\left\{A(i): i<o^{\vec{U}}(\kappa)\right\}$. Then $B$ is in $\bigcap\left\{U(\kappa, i): i<o^{\vec{U}}(\kappa)\right\}$.

Suppose for a contradiction that $\langle B\rangle$ does not have a direct extension which decides $\varphi$. Let $q=\left\langle d_{1}, \ldots, d_{n}, C\right\rangle$ be an extension of $\langle B\rangle$ which decides $\varphi$ such that $n$ is minimal. By assumption, $n>0$. Fix $l<2$ such that $q$ forces ${ }^{l} \varphi$. We will define a set $D \subseteq C$ such that $\left\langle d_{1}, \ldots, d_{n-1}, D\right\rangle$ forces ${ }^{l} \varphi$, which contradicts the minimality of $n$. Let $a_{1}, \ldots, a_{n}$ be the finite sequence from $P_{\kappa} \lambda$ which appears in $d_{1}, \ldots, d_{n}$. Let $\vec{d}=\left\langle d_{1}, \ldots, d_{n-1}\right\rangle$. Fix some $i$ such that $a_{n}$ is in $A(i)$.

Since $q$ forces ${ }^{l} \varphi, l\left(\vec{d}, a_{n}\right)=l$, and so for all $a$ in $A(i) \cap C, l(\vec{d}, a)=l$. So for each $a$ in $A(i) \cap C$ fix a condition $r_{a}=\left\langle\vec{d}, f_{a}, B_{a}\right\rangle$ below $\langle A\rangle$ which forces ${ }^{l} \varphi$ whose finite sequence from $P_{\kappa} \lambda$ is $a_{1}, \ldots, a_{n-1}, a$.

First suppose that $i=0$. Define $D=C \cap \triangle\left\{B_{a}: a \in C \cap A(i)\right\}$. Suppose for a contradiction that $q^{\prime}=\left\langle d_{1}, \ldots, d_{n-1}, D\right\rangle$ does not force ${ }^{l} \varphi$. Then there is a condition $r=\left\langle e_{1}, \ldots, e_{m}, E\right\rangle$ below $q^{\prime}$ which forces $\neg\left({ }^{l} \varphi\right)$. If $n>1$ then fix $k \leq m$ 
such that $e_{k}$ and $d_{n-1}$ have the same element from $P_{\kappa} \lambda$; if $n=1$ then let $k=0$ and $e_{k}=\emptyset$. If the least element $a$ of $r$ above $e_{k}$ satisfies that $o^{\vec{U}}(a \cap \kappa)=0$, then $a$ is in $C \cap A(0)$. Then $r_{a}$ and $r$ are compatible, which is impossible. Otherwise $o^{\vec{U}}(a \cap \kappa)>0$, so we can extend $r$ to $r^{\prime}$ by adding an element $a$ from $A(0) \cap C$ immediately above $e_{k}$. Then $r_{a}$ and $r^{\prime}$ are compatible, which is a contradiction.

Now assume that $i>0$. For each $a$ in $A(i) \cap C$, write $f_{a}=\left\langle a, b_{a}\right\rangle$, so $r_{a}=$ $\left\langle\vec{d},\left\langle a, b_{a}\right\rangle, B_{a}\right\rangle$.

We define three sets $D(<i), D(i)$, and $D(>i)$. Let $D(<i)$ be the set represented by the function $a \mapsto\left\{\pi_{a}{ }^{"} c: c \in b_{a}\right\}$ in $\operatorname{Ult}(V, U(\kappa, i))$. For all $a$ in $A(i) \cap C$, $\left\{\pi_{a}\right.$ " $\left.c: c \in b_{a}\right\}$ is in $\bigcap\left\{U(a \cap \kappa, \beta): \beta<o^{\vec{U}}(a \cap \kappa)\right\}$. Since $A(i) \cap C$ is in $U(\kappa, i)$, $D(<i)$ is in $\bigcap\{U(\kappa, \beta): \beta<i\}$. Also note that if $j: V \rightarrow \operatorname{Ult}(V, U(\kappa, i)), a \mapsto b_{a}$ represents $j$ " $D(<i)$.

Let $D(i)$ be the set of $a$ in $A(i) \cap C$ such that $D(<i) \cap P_{(a \cap \kappa)} a=b_{a}$.

Claim 5.6. The set $D(i)$ is in $U(\kappa, i)$.

Proof. Write $j: V \rightarrow \operatorname{Ult}(V, U(\kappa, i))$. Then by Lemma 1.1, $j(D(<i)) \cap P_{\kappa} j$ " $\lambda=$ $j$ " $D(<i)$. But $j$ " $D(<i)$ is represented by the function $a \mapsto b_{a}$ and $P_{\kappa} j$ " $\lambda$ is represented by the function $a \mapsto P_{(a \cap \kappa)} a$.

Finally, if $i$ is not the maximum ordinal below $o^{\vec{U}}(\kappa)$, then let $D(>i)$ be the set of $a$ in $\bigcup\left\{A(j): i<j<o^{\vec{U}}(\kappa)\right\}$ for which there exists $\beta<o^{\vec{U}}(a \cap \kappa)$ such that the set $\left\{\pi_{a}\right.$ "c $\left.c: c \in D(i) \cap P_{(a \cap \kappa)} a\right\}$ is in $U(a \cap \kappa, \beta)$. If $i$ is the maximum ordinal below $o^{\vec{U}}(\kappa)$ then let $D(>i)$ be the empty set.

Claim 5.7. The set $D(>i)$ is in $\bigcap\left\{U(\kappa, l): i<l<o^{\vec{U}}(\kappa)\right\}$.

Proof. Fix $l$ and let $j: V \rightarrow \operatorname{Ult}(V, U(\kappa, l))$. The set $D(i)$ is in $U(\kappa, i)$. But $D(i)$ is

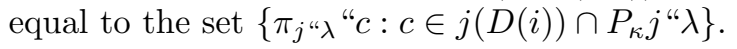

Let $D(<i)^{*}$ be the set of $a$ in $D(<i)$ such that $\left\{\pi_{a}\right.$ "c $\left.c: c \in D(<i) \cap P_{(a \cap \kappa)} a\right\}$ is in $\bigcap\left\{U(a \cap \kappa, l): l<o^{\vec{U}}(a \cap \kappa)\right\}$. Note that $D(<i)^{*}$ is in $\bigcap\{U(\kappa, l): l<i\}$.

Now define

$$
D=C \cap \triangle\left\{B_{a}: a \in C \cap A(i)\right\} \cap\left(D(<i)^{*} \cup D(i) \cup D(>i)\right) .
$$

Suppose for a contradiction that $q^{\prime}=\left\langle d_{1}, \ldots, d_{n-1}, D\right\rangle$ does not force ${ }^{l} \varphi$. Then there is a condition $r=\left\langle e_{1}, \ldots, e_{m}, E\right\rangle$ below $q^{\prime}$ which forces $\neg\left({ }^{l} \varphi\right)$. If $n>1$ then fix $k \leq m$ such that $e_{k}$ and $d_{n-1}$ have the same element from $P_{\kappa} \lambda$; if $n=1$ then let $k=0$ and $e_{k}=\emptyset$. Recall that for each $a$ in $A(i) \cap C, r_{a}=\left\langle d_{1}, \ldots, d_{n-1},\left\langle a, b_{a}\right\rangle, B_{a}\right\rangle$ is a condition which forces ${ }^{l} \varphi$. We will get a contradiction by showing that there is $a$ such that $r$ and $r_{a}$ are compatible. First note that we can extend each $r_{a}$ to $r_{a}^{\prime}$ whose finite sequence below $\left\langle a, b_{a}\right\rangle$ has length $k$ and has the same finite sequence from $P_{\kappa} \lambda$ as does $e_{1}, \ldots, e_{k}$.

We consider three cases. First suppose $k=m$. Extend $r$ to $r^{\prime}=\left\langle e_{1}, \ldots, e_{m}, e, E^{\prime}\right\rangle$ such that the element $a$ of $e$ from $P_{\kappa} \lambda$ is in $A(i)$. Then $r^{\prime}$ and $r_{a}^{\prime}$ have the same finite sequence of elements from $P_{\kappa} \lambda$ and so are compatible.

Now assume $k<m$. Suppose that for all $k<j \leq m$, the element of $e_{j}$ in $P_{\kappa} \lambda$ is in $D(<i)^{*}$. Fix $a$ in $D(i)$ such that $r^{\prime}=\left\langle e_{1}, \ldots, e_{m},\left\langle a, b_{a} \cap E\right\rangle, F\right\rangle \leq r$. By the definition of $D(i), b_{a}=D(<i) \cap P_{(a \cap \kappa)} a$. So the elements of $P_{\kappa} \lambda$ from $e_{k+1}, \ldots, e_{m}$ 
can be added to $r_{a}^{\prime}$ to obtain a condition with the same finite sequence as $r^{\prime}$. Then $r_{a}^{\prime}$ and $r^{\prime}$ are compatible.

Finally, suppose there is a minimum $j$ with $k<j \leq m$ such that $e_{j}=\left\langle a_{j}, B_{j}\right\rangle$ where $a_{j}$ is in $D(i) \cup D(>i)$. First assume that $a_{j}$ is in $D(i)$. For $k<l<j$, define $e_{l}^{*}$ by intersecting the measure one set appearing in $e_{l}$ (if it exists) with $D(<i)$. Then

$$
\left\langle e_{1}, \ldots, e_{k}, e_{k+1}^{*}, \ldots, e_{j-1}^{*},\left\langle a_{j}, B_{j} \cap b_{a_{j}}\right\rangle, e_{j+1}, \ldots, e_{m}, E \cap B_{a_{j}}\right\rangle
$$

is below $r_{a_{j}}^{\prime}$ and $r$. Otherwise the element $a_{j}$ of $e_{j}$ is in $D(>i)$. By the definition of $D(>i)$, we can extend $r$ by adding an element of $D(i)$ immediately below $e_{j}$. This condition satisfies that the least member $a$ of the finite sequence above $e_{k}$ which is in $D(i) \cup D(>i)$ is actually in $D(i)$, and we just proved that any such condition is compatible with $r_{a}^{\prime}$.

Now we can strengthen Sublemma 5.2.

Lemma 5.8. Suppose that $p=\left\langle d_{1}, \ldots, d_{n}, A\right\rangle$ is a condition in $\mathbb{R}$ and $m \leq n$ with $d_{m}=\left\langle a_{m}, A_{m}\right\rangle$. Let $G$ be generic for $\mathbb{R} / p^{\leq m}$. Then in $V[G]$ :

(1) for any $\varphi$ in the forcing language for $\mathbb{R}$ and any condition $s$ in $\mathbb{R} / p^{>m}$, there is $t \leq{ }^{*} s$ which decides $\varphi$.

(2) Suppose that $\xi<\kappa$ is an ordinal such that for all $l$ with $m<l \leq n$, if $d_{l}=\left\langle a_{l}, A_{l}\right\rangle$ where $o^{\vec{U}}\left(a_{l} \cap \kappa\right)>0$ then $\xi<a_{l} \cap \kappa$. Then for any collection $\left\{p_{i}: i<\xi\right\}$ of direct extensions of $p^{>m}$, there is $q$ such that $q \leq^{*} p_{i}$ for $i<\xi$.

(3) Suppose that $\xi<\kappa$ is an ordinal such that any a in $P_{\kappa} \lambda$ with $o^{\vec{U}}(a \cap \kappa)>0$ which appears either in the finite sequence of $p^{>m}$ or in a measure one set of $p^{>m}$ satisfies that $a \cap \kappa>\xi$. Then $p^{>m}$ forces that no new subsets of $\xi$ are added over $V[G]$.

Proof. The proof of (1) is similar to the proof of Sublemma 5.2, using Lemma 3.1 and the Prikry property for $\mathbb{R}$ in place of the assumption of Lemma 5.1. The proof of (2) is similar, using Lemma 3.1 .

For (3), every $r \leq p^{>m}$ satisfies the assumptions of (2). Suppose $q \leq p^{>m}$ and $q$ forces $\dot{f}: \xi \rightarrow 2$. For each $i<\xi$ let $q_{i} \leq^{*} q$ decides the value of $\dot{f}(i)$. Apply (2) and obtain $r$ such that $r \leq^{*} q_{i}$ for each $i$. Then $r$ forces that $\dot{f}$ is in the ground model.

\section{The Radin Club}

Suppose that $p$ is a condition in $\mathbb{R}$ and $a$ is in $P_{\kappa} \lambda$. We say that a appears in $p$ if either $a$ is in the finite sequence of $p$, or there is $d$ in the finite sequence of $p$ such that $d=\langle a, A\rangle$ for some $A$.

Suppose that $G$ is generic for $\mathbb{R}$ over $V$. Define the Radin club

$$
C_{G}=\left\{a \in\left(P_{\kappa} \lambda\right)^{V}: \exists p \in G(a \text { appears in } p)\right\} .
$$

By the compatibility of conditions in $G$, if $a$ and $b$ are in $C_{G}$, then either $a \subsetneq b$ or $b \precsim a$. Therefore either $a \cap \kappa<b \cap \kappa$ or vice versa. So $C_{G}$ is a well ordering and o.t. $\left(C_{G}\right) \leq \kappa$.

Proposition 6.1. The set $C_{G}$ is a closed and unbounded subset of $\left(P_{\kappa} \lambda\right)^{V}$. Furthermore, for all a in $C_{G}$ : 
(1) $a$ is a successor point of $C_{G}$ iff $o^{\vec{U}}(a \cap \kappa)=0$ and a is not equal to $\min \left(C_{G}\right)$.

(2) $a$ is a limit point of $C_{G}$ iff $o^{\vec{U}}(a \cap \kappa)>0$.

Proof. Suppose $a$ is in $C_{G}$ and $o^{\vec{U}}(a \cap \kappa)>0$. Fix $p$ in $G$ such that $a$ appears in $p$. Let $c$ be in $P_{(a \cap \kappa)} a$. Then there is a dense set of $r$ below $p$ in which there appears some $b$ with $c \underset{\sim}{\sim} \subsetneq a$. For if $q \leq p$, then $\langle a, A\rangle$ is in $q$ for some $A$, and it is possible to add elements $b$ from $A$ to $q$ with $c \underset{\sim}{\subseteq} \underset{\sim}{ }$. So $a$ is a limit point of $C_{G}$.

Suppose that $a$ is in $C_{G}, \vec{U}(a \cap \kappa)=0$, and $a$ is not equal to $\min \left(C_{G}\right)$. Fix $p$ in $G$ such that $a$ appears in $p$. It is easy to show that since $a$ is not minimal and

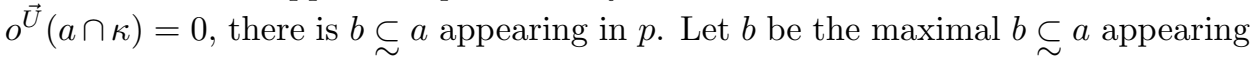

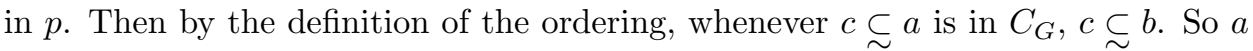
is the successor of $b$ in $C_{G}$.

The other directions of (1) and (2) now follow immediately.

An easy density argument shows that $C_{G}$ is unbounded in $\left(P_{\kappa} \lambda\right)^{V}$. Suppose for a contradiction that there is an initial segment $C$ of $C_{G}$ such that $\bigcup C=A$ is not in $C_{G}$. Let $a$ be the least element of $C_{G}$ such that $A \subset a$. Then $C_{G}$ is not unbounded in $P_{(a \cap \kappa)} a$, so $a$ is not a limit point of $C_{G}$. By (2) it follows that $o^{\vec{U}}(a \cap \kappa)=0$. By (1) there is $b$ in $C_{G}$ such that $a$ is the successor of $b$. But then every element of $C$ is a subset of $b$, so $A \subset b$. This contradicts the minimality of $a$.

The generic filter $G$ can be recovered from $C_{G}$. Let $p=\left\langle d_{1}, \ldots, d_{n}, A\right\rangle$ be a condition in $\mathbb{R}$ and let $a_{1}, \ldots, a_{n}$ be its finite sequence from $P_{\kappa} \lambda$. Then $p$ is in $G$ iff

(1) each $a_{i}$ is in $C_{G}$,

(2) for each $a$ in $C_{G}$ with $a_{n} \subsetneq a, a$ is in $A$ and the set $\left\{\pi_{a}{ }^{\prime \prime} c: c \in A \cap P_{(a \cap \kappa)} a\right\}$

is in $\bigcap\left\{U(a \cap \kappa, i): i<o^{\vec{U}}(a \cap \kappa)\right\}$, and

(3) write $a_{0}=\emptyset$; then for each $i \leq n$, if $d_{i}=\left\langle a_{i}, A_{i}\right\rangle$, then for all $a$ in $C_{G}$ with $a_{i-1} \cong a \subsetneq a_{i}, a$ is in $A_{i}$ and the set $\left\{\pi_{a}\right.$ " $\left.c: c \in A_{i} \cap P_{(a \cap \kappa)} a\right\}$ is in $\bigcap\{U(a \cap \kappa, i)$ :

$\left.i<o^{\vec{U}}(a \cap \kappa)\right\}$.

For any condition $p$ in $G$ satisfies these properties, and any condition which satisfies these conditions is compatible with each member of $G$, and so is in $G$.

We will abuse terminology and say that $C_{G}$ itself is generic for $\mathbb{R}$.

Lemma 6.2 (Factorization Lemma II). Suppose that $p=\left\langle d_{1}, \ldots, d_{n}, A\right\rangle$ is a condition in $\mathbb{R}, 0<m \leq n$, and $d_{m}=\left\langle a_{m}, A_{m}\right\rangle$.

Let $G$ be generic for $\mathbb{R}$ containing $p$. Define $C \leq m=\left\{\pi_{a_{m}}\right.$ "c $\left.c: c \in C_{G}, c \subseteq a_{m}\right\}$

and $C^{>m}=\left\{c \in C_{G}: a_{m} \subsetneq c\right\}$. Then $C^{\leq m}$ is generic for $\mathbb{R} / p^{\leq m}$ over $V$ and $C^{>m}$ is generic for $\mathbb{R} / p^{>m}$ over $V\left[C^{\leq m}\right]$.

On the other hand, if $C_{0}$ is generic for $\mathbb{R} / p^{\leq m}$ over $V$ and $C_{1}$ is generic for $\mathbb{R} / p^{>m}$ over $V\left[C_{0}\right]$, then $C=\left\{\pi_{a_{m}}^{-1} " c: c \in C_{0}\right\} \cup\left\{a_{m}\right\} \cup C_{1}$ is generic for $\mathbb{R} / p$ over $V$.

Proof. Let $i: \mathbb{R} / p \rightarrow \mathbb{R} / p^{\leq m} \times p^{>m}$ be the isomorphism described in the proof of Lemma 4.1. Write $i " G=G_{0} \times G_{1}$. Then $C_{G_{0}}=C^{\leq m}$ and $C_{G_{1}}=C^{>m}$. 
For the second statement, let $G=i^{-1 "}\left(G_{C_{0}} \times G_{C_{1}}\right)$. Then $C_{G}=C$.

Lemma 6.3. Suppose that $p=\left\langle d_{1}, \ldots, d_{n}, A\right\rangle$ is a condition in $\mathbb{R}, 0<m \leq n$, and $d_{m}=\left\langle a_{m}, A_{m}\right\rangle$. Let $G$ be generic for $\mathbb{R}$ containing $p$ and factor $C_{G}$ as $\bar{C} \leq m$ and $C^{>m}$. Then every subset of $\lambda_{\left(a_{m} \cap \kappa\right)}$ in $V[G]$ is in $V[C \leq m]$.

Proof. The condition $p^{>m}$ satisfies the property that for any $a$ in $P_{\kappa} \lambda$ appearing either in the finite sequence of $p^{>m}$ or in a measure one set of $p^{>m}, a \cap \kappa>\lambda_{a_{m} \cap \kappa}$. Now apply Lemma 5.8(3).

If $0<o^{\vec{U}}(\kappa)<\kappa$ then $\mathbb{R}$ is very similar to Magidor forcing, as we see next.

Lemma 6.4. (1) If the set $\left\{a \in C_{G}: o^{\vec{U}}(a \cap \kappa) \geq o^{\vec{U}}(\kappa)\right\}$ is non-empty, then it has a maximal element.

(2) For any $b$ in $C_{G}$, if the set $\left\{a \in C_{G}: a \subsetneq b, o^{\vec{U}}(a \cap \kappa) \geq o^{\vec{U}}(b \cap \kappa)\right\}$ is non-empty, then it has a maximal element.

Proof. Let $C=\left\{a \in C_{G}: o^{\vec{U}}(a \cap \kappa) \geq o^{\vec{U}}(\kappa)\right\}$. If $C$ is empty then we are done. Suppose that $C$ is non-empty. Fix $p=\left\langle d_{1}, \ldots, d_{n}, A\right\rangle$ in $G$ such that there is $0<m \leq n$ with $d_{m}=\left\langle a_{m}, A_{m}\right\rangle$ and $a_{m}$ is the largest element of $C$ appearing in $p$. Define $q \leq p$ by replacing $A$ with $A^{\prime}=\left\{a \in A: o^{\vec{U}}(a \cap \kappa)<o^{\vec{U}}(\kappa)\right\}$. Then $q$ forces that any $c$ in $C_{G}$ with $o^{\vec{U}}(c \cap \kappa) \geq o^{\vec{U}}(\kappa)$ is a subset of $b$. Therefore $q$ forces that $a$ is the maximal element of $C$.

Statement (2) follows from the first statement applied to the Radin forcing $\mathbb{R}(\vec{U} \uparrow$ $((b \cap \kappa)+1))$.

Theorem 6.5. Suppose that $0<o^{\vec{U}}(\kappa)<\kappa$. If there is a in $C_{G}$ such that $\vec{U}(a \cap$ $\kappa) \geq o^{\vec{U}}(\kappa)$, then let $a^{\prime}$ be the maximal such set. Otherwise let $a^{\prime}=\emptyset$. Then the set $\left\{b \in C_{G}: a^{\prime} \subsetneq b\right\}$ is a closed and unbounded subset of $\left(P_{\kappa} \lambda\right)^{V}$ with order type $\omega^{o^{\vec{U}}}(\kappa)$.

Proof. We prove the theorem by induction on $o^{\vec{U}}(\kappa)$. Let $C_{G}^{\prime}=\left\{a \in C_{G}: a^{\prime} \subsetneq a\right\}$.

Suppose that $o^{\vec{U}}(\kappa)=1$. Then for all $b$ in $C_{G}^{\prime}, o^{\vec{U}}(b \cap \kappa)=0$. By Proposition $6.1(2), C_{G}^{\prime}$ has no limits points. So the order type of $C_{G}^{\prime}$ is $\omega$.

Suppose that $o^{\vec{U}}(\kappa)=\xi+1$ and the statement holds for ordinals less than $\xi+1$. Then for all $b$ in $C_{G}^{\prime}, o^{\vec{U}}(b \cap \kappa) \leq \xi$. By a density argument the set $C_{\xi}=\left\{a \in C_{G}^{\prime}: o^{\vec{U}}(a \cap \kappa)=\xi\right\}$ is unbounded in $C_{G}^{\prime}$. By Proposition $6.4(2), C_{\xi}$ has no limit points in $C_{G}^{\prime}$ and so has order type $\omega$. Let $\left\langle a_{n}: 0<n<\omega\right\rangle$ enumerate the elements of $C_{\xi}$ in increasing order and let $a_{0}=a^{\prime}$. By the the induction hypothesis and Lemma 6.2, for each $n \geq 1$ the set $C_{n}=\left\{b \in C_{G}^{\prime}: a_{n-1} \subsetneq b \subsetneq a_{n}\right\}$ has order type $\omega^{\xi}$. Thus $C_{G}^{\prime}$ has order type $\omega^{\xi} \cdot \omega=\omega^{\xi+1}$.

Now suppose that $o^{\vec{U}}(\kappa)$ is a limit ordinal and the statement holds for all $\delta<$ $o^{\vec{U}}(\kappa)$. Fix $\delta<o^{\vec{U}}(\kappa)$. By a density argument, there is a set $b$ in $C_{G}^{\prime}$ with $o^{\vec{U}}(b \cap \kappa)=$ $\delta$. So by the induction hypothesis and Lemma $6.2, C_{G}^{\prime}$ has order type at least $\omega^{\delta}$. Therefore $C_{G}^{\prime}$ has order type at least $\sup \left\{\omega^{\delta}: \delta<o^{\vec{U}}(\kappa)\right\}=\omega^{o^{\vec{U}}(\kappa)}$. Suppose for a contradiction that the order type of $C_{G}^{\prime}$ is larger than $\omega^{o^{\vec{U}}(\kappa)}$. Let $b$ be the 
$\omega^{o^{\vec{U}}(\kappa)}$-th element of $C_{G}^{\prime}$. By the induction hypothesis and Lemma 6.2 , there is a tail of $C_{G}^{\prime} \cap P_{(b \cap \kappa)} b$ with order type $\omega^{o^{\vec{V}}(b \cap \kappa)}$. But every tail of $C_{G}^{\prime} \cap P_{(b \cap \kappa)} b$ has order type $\omega^{o^{\vec{U}}(\kappa)}$, which is a contradiction.

Corollary 6.6. Suppose that $0<o^{\vec{U}}(\kappa)<\kappa$. Then there is $p \leq^{*} 1$ which forces that the order type of $C_{G}$ is $\omega^{o^{\vec{U}}(\kappa)}$.

Proof. Let $p=\langle A\rangle$, where $A$ is the set of $a$ in $A_{\vec{U}}$ with $o^{\vec{U}}(a \cap \kappa)<o^{\vec{U}}(\kappa)$.

Note that if $o^{\vec{U}}(\alpha)<o^{\vec{U}}(\kappa)<\kappa$ for all $\alpha<\kappa$, then the order type of $C_{G}$ is $\omega^{o^{\vec{U}}(\kappa)}$.

\section{Preservation of Cardinals}

When $\lambda=\kappa$ then $\mathbb{R}$ preserves all cardinals. When $\lambda>\kappa$ the situation is a bit more complicated, but we have a description of which cardinals are collapsed.

For each $a$ in $P_{\kappa} \lambda$ write $\kappa_{a}=a \cap \kappa$. Let $G$ be generic for $\mathbb{R}$ over $V$ and let $C_{G}$ be the Radin club described in the last section.

Recall that since $\kappa$ is $\lambda$-supercompact, $\lambda^{<\kappa}$ is equal to $\lambda$ if $\operatorname{cf}(\lambda) \geq \kappa$ and is equal to $\lambda^{+}$if $\operatorname{cf}(\lambda)<\kappa$.

Proposition 7.1. Let $\mu \geq \kappa$ be a cardinal.

(1) All cardinals greater than $\lambda^{<\kappa}$ are preserved.

(2) If $\mu \leq \lambda$ and $\operatorname{cf}(\mu) \geq \kappa$, then in $V[G], \operatorname{cf}(\mu)=\operatorname{cf}\left(\right.$ o.t. $\left.\left(C_{G}\right)\right)$.

(3) If $\kappa<\mu \leq \lambda^{<\kappa}$ then $\mu$ is collapsed in $V[G]$.

Proof. Statement (1) follows from the fact that $\mathbb{R}$ is $\left(\lambda^{<\kappa}\right)^{+}$-c.c. Suppose that $\kappa \leq \mu \leq \lambda$ and $\operatorname{cf}(\mu) \geq \kappa$ in $V$. By a density argument the set $\left\{\sup (a \cap \mu): a \in C_{G}\right\}$ is closed and unbounded in $\mu$ with order type o.t. $\left(C_{G}\right)$. So $\operatorname{cf}(\mu)=\operatorname{cf}\left(\right.$ o.t. $\left.\left(C_{G}\right)\right)$. Since o.t. $\left(C_{G}\right) \leq \kappa$, every successor cardinal in the interval $(\kappa, \lambda]$ is collapsed. It follows that every cardinal in this interval is collapsed.

If $\operatorname{cf}(\lambda) \geq \kappa$, then $\lambda^{<\kappa}=\lambda$ and the proof is complete. If $\operatorname{cf}(\lambda)<\kappa$ then $\lambda^{<\kappa}=\lambda^{+}$. Every member of $\left(P_{\kappa} \lambda\right)^{V}$ is a subset of some $a$ in $C_{G}$, and for any $a$ in $C_{G}$ there are fewer than $\kappa$ many subsets of $a$ in $V$. It follows that in $V[G]$ the ordinal $\left(\lambda^{<\kappa}\right)^{V}$ has size no larger than $\left|C_{G}\right| \cdot \kappa=\kappa$.

Proposition 7.2. Let $\mu<\kappa$ be a cardinal.

(1) The cardinal $\mu$ is collapsed iff there is a in $C_{G}$ with $o^{\vec{U}}\left(\kappa_{a}\right)>0$ such that $\kappa_{a}<\mu \leq \lambda_{\kappa_{a}}^{<\kappa_{a}}$.

(2) Suppose there is a in $C_{G}$ with $O^{\vec{U}}\left(\kappa_{a}\right)>0$ such that $\kappa_{a} \leq \mu \leq \lambda_{\kappa_{a}}$ and $\operatorname{cf}(\mu) \geq \kappa_{a}$. Then in $V[G], \operatorname{cf}(\mu)=\operatorname{cf}\left(\right.$ o.t. $\left.\left(C_{G} \cap P_{\kappa_{a}} a\right)\right)$.

(3) If $\mu$ is regular and $\mu$ changes its cofinality, then there is a in $C_{G}$ with $\vec{U}\left(\kappa_{a}\right)>0$ such that $\kappa_{a} \leq \mu \leq \lambda_{\kappa_{a}}^{<\kappa_{a}}$.

Proof. Suppose that $a$ is in $C_{G}$ with $o^{\vec{U}}\left(\kappa_{a}\right)>0$ and $\kappa_{a}<\mu \leq \lambda_{\kappa_{a}}^{<\kappa_{a}}$. Then we can factor $C_{G}$ as $C_{0}$ and $C_{1}$ such that $C_{0}$ is generic for $\mathbb{R}\left(\vec{U} \uparrow\left(\kappa_{a}+1\right)\right)$ over $V$. By Proposition 7.1(3), $\mu$ is collapsed in $V\left[C_{0}\right]$ and so also in $V[G]$. By Proposition 7.1(2), if $\mu \leq \lambda_{\kappa_{a}}$ and $\operatorname{cf}(\mu) \geq \kappa_{a}$, then in $V\left[C_{0}\right], \operatorname{cf}(\mu)=\operatorname{cf}\left(\right.$ o.t. $\left.\left(C_{0}\right)\right)=\operatorname{cf}\left(\right.$ o.t. $\left(C_{G} \cap\right.$ $\left.P_{\kappa_{a}} a\right)$ ). By Lemma 6.3, the same is true in $V[G]$. This proves (2) and the "if" direction of (1). 
JOHN KRUEGER

(3) Suppose that $\mu$ is regular, $\beta<\mu, p=\left\langle d_{1}, \ldots, d_{n}, A\right\rangle$ is in $G$, and $p$ forces that $\dot{f}: \beta \rightarrow \mu$ is cofinal. Assume for a contradiction that there is no $q \leq p$ which forces the conclusion of (3). Then in particular, if $0<i \leq n$ and $d_{i}=\left\langle a_{i}, A_{i}\right\rangle$, then $\mu$ is not in the interval $\left[\kappa_{a_{i}}, \lambda_{a_{i}}^{<\kappa_{a_{i}}}\right]$. Define $V_{1}$ and $p^{*}$ as follows. If there is a largest $m \leq n$ such that $o^{\vec{U}}\left(\kappa_{a_{m}}\right)>0$ and $\mu>\kappa_{a_{m}}$, let $V_{1}$ be $V[G \leq m]$, where $G^{\leq m}$ is the generic for $\mathbb{R} / p^{\leq m}$ given by $G$, and let $p^{*}=p^{>m}$; otherwise let $V_{1}=V$ and $p^{*}=p$. In either case, $\mu$ is regular in $V_{1}$, and for any $a$ appearing in $p^{*}$ with $o^{\vec{U}}\left(\kappa_{a}\right)>0, \mu<\kappa_{a}$. For each pair $\langle i, \alpha\rangle$ in $\beta \times \mu$, choose $q(i, \alpha) \leq^{*} p^{*}$ which decides the statement $\dot{f}(i)=\alpha$. By Lemmas 3.1 and 5.8(2), there is $q$ such that $q \leq^{*} q(i, \alpha)$ for each $i$ and $\alpha$. Define $g: \beta \rightarrow \mu$ in $V_{1}$ by letting $g(i)$ be such that $q$ forces $\dot{f}(i)=g(i)$. Then $g$ is cofinal in $\mu$ and therefore $\mu$ is singular in $V_{1}$, which is a contradiction.

"Only if" direction of (1): First we show that if $\mu$ is collapsed, then there is $a$ in $C_{G}$ with $o^{\vec{U}}\left(\kappa_{a}\right)>0$ such that $\kappa_{a} \leq \mu \leq \lambda_{\kappa_{a}}^{<\kappa_{a}}$. If $\mu$ is regular then this follows from (3). Suppose $\mu$ is singular. Then there is a sequence $\left\langle\alpha_{i}: i<\operatorname{cf}(\mu)\right\rangle$ of regular cardinals unbounded in $\mu$ in $V$ which are all collapsed in $V[G]$. For each $i$ fix $a_{i}$ in $C_{G}$ such that $\kappa_{a_{i}} \leq \alpha_{i} \leq \lambda_{\kappa_{a_{i}}}^{<\kappa_{a_{i}}}$. If the map $i \mapsto a_{i}$ is not eventually constant, then $\mu$ is a limit point of the club set $\left\{a \cap \kappa: a \in C_{G}\right\}$, and so is in this set. This is a contradiction since $\mu$ is singular in $V$, and if $a$ is a limit point of $C_{G}$ then $a \cap \kappa$ is measurable in $V$. If $a$ is in $C_{G}$ such that $a_{i}=a$ for a tail of $i$ 's, then $\kappa_{a} \leq \mu \leq \lambda_{\kappa_{a}}^{<\kappa_{a}}$.

Now suppose that $\mu=\kappa_{a}$ for some $a$ in $C_{G}$ with $o^{\vec{U}}\left(\kappa_{a}\right)>0$. We show that $\mu$ is not collapsed. In $V[G]$ the cardinal $\mu$ is a limit of point of $\left\{\kappa_{b}: b \in C_{G}, o^{\vec{U}}(b \cap \kappa)=\right.$ $0\}$, and by the last paragraph every ordinal in this set is a cardinal in $V[G]$.

By Proposition 7.2(1), the set $\left\{\kappa_{a}: a \in C_{G}\right\}$ is a club subset of $\kappa$ consisting of cardinals. So $\kappa$ remains a cardinal in $V[G]$.

By the theorem of Cummings and Shelah about changes of cofinality (see Section 1 ) and the fact that $\mathbb{R}$ is $\left(\lambda^{<\kappa}\right)^{+}$-c.c., the cofinality of $\lambda^{<\kappa}$ in $V[G]$ is equal to $\operatorname{cf}\left(\left|\left(\lambda^{<\kappa}\right)^{V}\right|\right)=\operatorname{cf}(\kappa)=\operatorname{cf}\left(\right.$ o.t. $\left.\left(C_{G}\right)\right)$. Similarly, if $a$ is in $C_{G}$ then in $V[G]$ the cofinality of $\lambda_{\kappa_{a}}^{<\kappa_{a}}$ is equal to $\operatorname{cf}\left(\right.$ o.t. $\left.\left(C_{G} \cap P_{(a \cap \kappa)} a\right)\right)$.

\section{PART II: ITERATED RADIN FORCING}

\section{ITERATING RADIN FORCING OVER THE SAME CARDINAL}

In this section we show how to iterate Radin forcing infinitely many times over the same cardinal. Suppose that $\kappa$ is a supercompact cardinal. Let $\left\langle\kappa_{n}: n\langle\omega\rangle\right.$ be a sequence of cardinals such that either (1) each $\kappa_{n}$ is equal to $\kappa$, or (2) $\kappa_{0} \geq \kappa$ and $\kappa_{n}<\left(\kappa_{n}^{<\kappa}\right)^{+} \leq \kappa_{n+1}$ for each $n$. We will define a forcing iteration $\mathbb{R}^{\omega}$ with length $\omega$ such that at each stage $n$ we force with a Radin forcing defined from a carefully chosen coherent sequence of ultrafilters on $P_{\kappa} \kappa_{n}$.

Define by recursion sequences $\left\langle\mathbb{R}_{n}: n<\omega\right\rangle$ and $\left\langle\mathbb{R}^{n}: n<\omega\right\rangle$ as follows. We let $\mathbb{R}^{0}$ be the trivial poset and for $n>0$ let $\mathbb{R}^{n}=\mathbb{R}_{0} * \ldots * \mathbb{R}_{n-1}$. We maintain the following for each $n \geq 0$ : 


\section{Recursion Hypotheses}

(1) $\mathbb{R}^{n}$ forces that $\kappa$ is supercompact, and

(2) if $n>0$ then $\left|\mathbb{R}^{n}\right| \leq 2^{\left(\kappa_{n-1}^{<\kappa}\right)}$ and $\mathbb{R}^{n}$ is $\left(\kappa_{n-1}^{<\kappa}\right)^{+}$-c.c.

Obviously $\mathbb{R}^{0}$ forces that $\kappa$ is supercompact. Suppose that $n \geq 0$ and $\mathbb{R}^{n}$ is defined and satisfies the recursion hypotheses. Let $G^{n}$ be generic for $\mathbb{R}^{n}$ over $V$ and write $V_{n}=V\left[G^{n}\right]$.

We would like to define a Radin forcing $\mathbb{R}_{n}$ on $P_{\kappa} \kappa_{n}$ in $V_{n}$ which forces that $\kappa$ remains supercompact. In order to achieve this we must choose our coherent sequence carefully. Until further notice we are working in the model $V_{n}$. We begin with a variation of Proposition 2.2.

Proposition 8.1. Suppose that $\lambda \geq 2^{\left(\kappa_{n}^{<\kappa}\right)}, U$ is a normal ultrafilter on $P_{\kappa} \lambda$ with $j: V \rightarrow M=\operatorname{Ult}(V, U)$, and there is a function $f: \kappa \rightarrow \kappa$ such that $j(f)(\kappa)=\kappa_{n}$. Then there exists a coherent sequence of ultrafilters $\vec{U}=\left\langle U(\alpha, i): \alpha \leq \kappa, i<o^{\vec{U}}(\alpha)\right\rangle$ on $P_{\kappa} \kappa_{n}$ such that:

(1) $0<o^{\vec{U}}(\kappa)<\left(2^{\left(\kappa_{n}^{<\kappa}\right)}\right)^{+}$,

(2) $j(\vec{U}) \uparrow(\kappa+1)=\vec{U}$, and

(3) $\bigcap\left\{U(\kappa, i): i<o^{\vec{U}}(\kappa)\right\}$ is a subset of $U \uparrow \kappa_{n}$.

Proof. Define $g: \kappa \rightarrow V_{\kappa}$ recursively as follows. The domain of $g$ is the set of strongly inaccessible cardinals $\alpha$ such that $f(\alpha) \geq \alpha$ is a cardinal and $\alpha$ is closed under $f$. Suppose that $\alpha$ is in the domain of $g$ and $g\left\lceil\alpha\right.$ is defined. Let $X_{\alpha}$ be the collection of sequences $\left\langle U_{i}: i\langle\gamma\rangle\right.$ of normal ultrafilters on $P_{\alpha} f(\alpha)$ such that for all $\beta<\gamma$, letting $j_{\beta}: V \rightarrow \operatorname{Ult}\left(V, U_{\beta}\right), j_{\beta}\left(g\lceil\alpha)(\alpha)=\left\langle U_{i}: i<\beta\right\rangle\right.$; moreover, for each $\beta<\gamma, U_{\beta} \backslash \bigcup\left\{U_{i}: i<\beta\right\}$ is non-empty. Let $g(\alpha)=\left\langle U(\alpha, i): i<o^{\vec{U}}(\alpha)\right\rangle$ be some maximal element in $X_{\alpha}$, which exists by Zorn's Lemma.

Let $\left\langle U(\kappa, i): i<o^{\vec{U}}(\kappa)\right\rangle$ be equal to $j(g)(\kappa)$. This defines a coherent sequence $\vec{U}$ on $P_{\kappa} \kappa_{n}$ such that $j(\vec{U}) \uparrow(\kappa+1)=\vec{U}$.

Note that $o^{\vec{U}}(\kappa)<\left(2^{\left(\kappa_{n}^{<\kappa}\right)}\right)^{+}$. For otherwise choose $X_{i}$ in $U(\kappa, i) \backslash \bigcup\{U(\kappa, j)$ : $j<i$ \} for each $i<\left(2^{\left(\kappa_{n}^{<\kappa}\right)}\right)^{+}$. Then $X_{i} \neq X_{j}$ for $i<j$, since $X_{j}$ is not in $U(\kappa, i)$. This is a contradiction since there are only $2^{\left(\kappa_{n}^{<\kappa}\right)}$ many subsets of $P_{\kappa} \kappa_{n}$.

Let $U^{\prime}=U \uparrow \kappa_{n}$ and write $i: V \rightarrow N=\operatorname{Ult}\left(V_{n}, U^{\prime}\right)$. Let $k: N \rightarrow M$ be the elementary embedding from Lemma 1.3 such that $j=k \circ i$ and $\operatorname{crit}(k)=\left(2^{\left(\kappa_{n}^{<\kappa}\right)}\right)^{+N}$. Now $k$ maps the critical point of $k$ to $\left(2^{\left(\kappa_{n}^{<\kappa}\right)}\right)^{+}$, which is larger than $o^{\vec{U}}(\kappa)$, so $i(g)(\kappa)$ has length $o^{\vec{U}}(\kappa)$ and in fact is equal to $\left\langle U(\kappa, i): i<o^{\vec{U}}(\kappa)\right\rangle$. Since $U^{\prime}$ is in $M$, by the maximality of $j(g)(\kappa), U^{\prime} \backslash \bigcup\left\{U(\kappa, i): i<o^{\vec{U}}(\kappa)\right\}$ is empty. Suppose that $A$ is in $\bigcap\left\{U(\kappa, i): i<o^{\vec{U}}(\kappa)\right\}$. If $A$ is not in $U^{\prime}$, then $\left(P_{\kappa} \kappa_{n} \backslash A\right)$ is in $U^{\prime}$. So there is $i<o^{\vec{U}}(\kappa)$ such that $\left(P_{\kappa} \kappa_{n} \backslash A\right)$ and $A$ are both in $U(\kappa, i)$, which is impossible.

Lemma 8.2. There exists a coherent sequence $\vec{U}=\left\langle U(\alpha, i): \alpha \leq \kappa, i<o^{\vec{U}}(\alpha)\right\rangle$ of ultrafilters on $P_{\kappa} \kappa_{n}$ in $V_{n}$ with $0<o^{\vec{U}}(\kappa)<\left(2^{\left(\kappa_{n}^{<\kappa}\right)}\right)^{+}$satisfying the following property: for every $\lambda \geq 2^{\left(\kappa_{n}^{<\kappa}\right)}$ there is a normal ultrafilter $U_{\lambda}$ on $P_{\kappa} \lambda$ with $j_{\lambda}$ : $V_{n} \rightarrow M_{\lambda}=\operatorname{Ult}\left(V, U_{\lambda}\right)$ such that $j_{\lambda}(\vec{U}) \uparrow(\kappa+1)=\vec{U}$ and $\bigcap\left\{U(\kappa, i): i<o^{\vec{U}}(\kappa)\right\} \subseteq$ $U_{\lambda}\left\lceil\kappa_{n}\right.$. 
Proof. By Lemma 1.3 it suffices to prove there are unboundedly many regular cardinals $\lambda$ satisfying the required property. Suppose for a contradiction that for each coherent sequence $\vec{W}$ on $P_{\kappa} \kappa_{n}$ with $o^{\vec{W}}(\kappa)<\left(2^{\left(\kappa_{n}^{<\kappa}\right)}\right)^{+}$, there is $\mu(\vec{W}) \geq 2^{\left(\kappa_{n}^{<\kappa}\right)}$ such that for every regular $\lambda \geq \mu(\vec{W})$, there is no $U_{\lambda}$ on $P_{\kappa} \lambda$ as desired. Choose a regular cardinal $\lambda$ which is larger than $\mu(\vec{W})$ for each coherent sequence $\vec{W}$.

Let $f: \kappa \rightarrow V_{\kappa}$ be a Laver function for $\kappa$. Then there exists a normal ultrafilter $U$ on $P_{\kappa} \lambda$ with elementary embedding $j: V_{n} \rightarrow M=\operatorname{Ult}\left(V_{n}, U\right)$ such that $j(f)(\kappa)=$ $\kappa_{n}$. Apply Proposition 8.1 to obtain $\vec{U}$, which contradicts that $\lambda$ is greater than $\mu(\vec{U})$.

Now let $\mathbb{R}_{n}$ be an $\mathbb{R}^{n}$-name for a Radin forcing defined from a coherent sequence on $P_{\kappa} \kappa_{n}$ satisfying the statement of Lemma 8.2. Define $\mathbb{R}^{n+1}=\mathbb{R}^{n} * \mathbb{R}_{n}$.

Proposition 8.3. The poset $\mathbb{R}^{n+1}$ forces that $\kappa$ is supercompact.

Proof. Suppose that $G^{n} * G_{n}$ is generic for $\mathbb{R}^{n+1}=\mathbb{R}^{n} * \mathbb{R}_{n}$ over $V$ and write $V_{n}=V\left[G^{n}\right]$. Let $\vec{U}=\left\langle U(\alpha, i): \alpha \leq \kappa, i<o^{\vec{U}}(\alpha)\right\rangle$ be the coherent sequence on $P_{\kappa} \kappa_{n}$ in $V_{n}$ such that $\mathbb{R}_{n}=\mathbb{R}(\vec{U})$. Fix $\lambda \geq 2^{\left(\kappa_{n}^{<\kappa}\right)}$. Let $U$ be a normal ultrafilter on $P_{\kappa} \lambda$ with $j: V_{n} \rightarrow M=\operatorname{Ult}(V, U)$ such that $j(\vec{U})\lceil(\kappa+1)=\vec{U}$ and $\bigcap\{U(\kappa, i)$ : $\left.i<o^{\vec{U}}(\kappa)\right\} \subseteq U\left\lceil\kappa_{n}\right.$.

Define $A^{*}$ to be the set of $a$ in $j\left(A_{\vec{U}}\right)$ such that $j$ " $\kappa_{n} \subsetneq a$. Recall that in $M$, $\pi_{\left(j " \kappa_{n}\right)}=j^{-1} \uparrow j$ “" $\kappa_{n}$. Let $s$ be the condition

$$
\left\langle\left\langle j “ \kappa_{n}, j " A_{\vec{U}}\right\rangle, A^{*}\right\rangle
$$

in $j\left(\mathbb{R}_{n}\right)$. Then $j\left(\mathbb{R}_{n}\right) / s$ factors as $\mathbb{R}_{n} \times j\left(\mathbb{R}_{n}\right) /\left\langle A^{*}\right\rangle$ with isomorphism $i$ as described in the proof of Lemma 4.1.

For any $p=\left\langle d_{1}, \ldots, d_{n}, A\right\rangle$ in $\mathbb{R}_{n}, j(p)=\left\langle j\left(d_{1}\right), \ldots, j\left(d_{n}\right), j(A)\right\rangle$. Let

$$
p^{\prime}=\left\langle j\left(d_{1}\right), \ldots, j\left(d_{n}\right),\left\langle j{ }^{*} \kappa_{n}, j " A\right\rangle, j(A) \cap A^{*}\right\rangle .
$$

Since $A$ is in $\bigcap\left\{U(\kappa, i): i<o^{\vec{U}}(\kappa)\right\rangle, A$ is in $U \uparrow \kappa_{n}$. Therefore $j " \kappa_{n} \in j(A)$. It follows that $p^{\prime}$ is below $j(p)$. Clearly $p^{\prime}$ is below $s$ as well. Moreover, $i\left(p^{\prime}\right)=$ $\left\langle p,\left\langle j(A) \cap A^{*}\right\rangle\right\rangle$.

Define $U^{*}$ in $V_{n}\left[G_{n}\right]$ by letting $X$ be in $U^{*}$ iff $X \subseteq P_{\kappa} \lambda$, there is a name $\dot{X}$ for $X$, and there is $t \leq^{*}\left\langle A^{*}\right\rangle$ in $j\left(\mathbb{R}_{n}\right) /\left\langle A^{*}\right\rangle$ such that $t$ forces over $M\left[G_{n}\right]$ that $j^{\text {" }} \lambda$ is in $j(\dot{X})$.

First we show that the definition does not depend on the choice of the name $\dot{X}$. Suppose that $X=\dot{X}^{G_{n}}=\dot{Y}^{G_{n}}$ in $V_{n}\left[G_{n}\right]$. Then there is $p=\left\langle d_{1}, \ldots, d_{n}, A\right\rangle$ in $G_{n}$ which forces that $\dot{X}=\dot{Y}$. Since $p^{\prime} \leq j(p), p^{\prime}$ forces over $M$ that $j(\dot{X})=j(\dot{Y})$. But $i\left(p^{\prime}\right)=\left\langle p,\left\langle j(A) \cap A^{*}\right\rangle\right\rangle$, so $\left\langle j(A) \cap A^{*}\right\rangle$ forces over $M\left[G_{n}\right]$ that $j(\dot{X})=j(\dot{Y})$. Applying the Prikry property of $\mathbb{R} /\left\langle A^{*}\right\rangle$ in $M\left[G_{n}\right]$, let $t_{\dot{X}}$ (respectively, $t_{\dot{Y}}$ ) be a direct extension of $\left\langle A^{*}\right\rangle$ which decides whether $j$ " $\lambda$ is in $j(\dot{X})$ (respectively, in $j(\dot{Y}))$. But $t_{\dot{X}}, t_{\dot{Y}}$, and $\left\langle j(A) \cap A^{*}\right\rangle$ are compatible, so $t_{\dot{X}}$ and $t_{\dot{Y}}$ must decide their respective statements the same way.

Similar arguments show that $U^{*}$ is a non-principal, fine ultrafilter on $P_{\kappa} \lambda$ which extends $U$. To prove normality, suppose that $p=\left\langle d_{1}, \ldots, d_{n}, A\right\rangle$ is in $G_{n}$ and $p$ forces that $\dot{f}: P_{\kappa} \lambda \rightarrow \lambda$ is a regressive function. Then $\left\langle j(A) \cap A^{*}\right\rangle$ forces over $M\left[G_{n}\right]$ that $j(\dot{f}): j\left(P_{\kappa} \lambda\right) \rightarrow j(\lambda)$ is a regressive function. For each $i$ less than $\lambda$ let $t_{i}$ be a direct extension of $\left\langle j(A) \cap A^{*}\right\rangle$ which decides whether $j(\dot{f})\left(j^{\prime \prime} \lambda\right)=j(i)$. 
By Lemma 5.8 there is $t$ such that $t \leq^{*} t_{i}$ for all $i$. Clearly there must exist $i$ such that $t$ forces that $j(\dot{f})(j “ \lambda)=j(i)$. Then the set $\left\{a \in P_{\kappa} \lambda: f(a)=i\right\}$ is in $U^{*}$.

This proves the first recursion hypothesis. If $n=0$ then $\mathbb{R}^{1}=\mathbb{R}_{0}$, which has size at most $2^{\left(\kappa_{0}^{<\kappa}\right)}$ and is $\left(\kappa_{0}^{<\kappa}\right)^{+}$-c.c. Suppose that $n>0$. Then by recursion $\mathbb{R}^{n}$ has size at most $2^{\left(\kappa_{n-1}^{<\kappa}\right)}$ and is $\left(\kappa_{n-1}^{<\kappa}\right)^{+}$-c.c.

Lemma 8.4. Suppose that $G^{n}$ is generic for $\mathbb{R}^{n}$ over $V$. Then the cardinals $\kappa_{n}^{<\kappa}$ and $2^{\left(\kappa_{n}^{<\kappa}\right)}$ are computed the same in both $V$ and $V\left[G^{n}\right]$.

Proof. First assume that $\operatorname{cf}\left(\kappa_{n}\right)<\kappa$ in $V$. Since $\kappa$ is supercompact in $V$ and $V\left[G^{n}\right], \kappa_{n}^{<\kappa}=\kappa_{n}^{+}$in both models. But $\mathbb{R}^{n}$ is $\kappa_{n}$-c.c. Now suppose that $\operatorname{cf}\left(\kappa_{n}\right) \geq \kappa$ in $V$. Then $\kappa_{n}^{<\kappa}=\kappa_{n}$ in $V$. If $\operatorname{cf}\left(\kappa_{n}\right)^{V}$ changes its cofinality in $V\left[G^{n}\right]$, then by Proposition 7.1 it must change its cofinality to $\kappa$. So $\kappa_{n}^{<\kappa}=\kappa_{n}$ in $V\left[G^{n}\right]$ as well.

The poset $\mathbb{R}^{n}$ has at most $2^{\left(\kappa_{n-1}^{<\kappa}\right)}$ many antichains. So there are at most $2^{\left(\kappa_{n}^{<\kappa}\right)}$ many canonical names for subsets of $\kappa_{n}^{<\kappa}$.

Since $\mathbb{R}^{n}$ is $\left(\kappa_{n-1}^{<\kappa}\right)^{+}$-c.c. and $\mathbb{R}^{n}$ forces that $\mathbb{R}_{n}$ is $\left(\kappa_{n}^{<\kappa}\right)^{+}$-c.c., $\mathbb{R}^{n+1}$ is $\left(\kappa_{n}^{<\kappa}\right)^{+}$c.c. The chain condition and sizes of $\mathbb{R}^{n}$ and $\mathbb{R}_{n}$, along with Lemma 8.4, imply that $\left|\mathbb{R}^{n+1}\right| \leq 2^{\left(\kappa_{n}^{<\kappa}\right)}$.

Now we define the iteration $\mathbb{R}^{\omega}$. The poset $\mathbb{R}^{\omega}$ is basically a Magidor iteration, except instead of iterating Prikry type forcing posets over different cardinals, we iterate Radin forcing over a fixed cardinal.

A condition in $\mathbb{R}^{\omega}$ is a function $p$ with domain $\omega$ such that for all $n<\omega, p \uparrow n$ is in $\mathbb{R}^{n}$; moreover, there is a finite set $a_{p} \subseteq \omega$ such that for all $n<\omega, n$ is in $\omega \backslash a_{p}$ iff $p\left\lceil n\right.$ forces that $p(n) \leq^{*} 1$. Let $q \leq p$ if $q \uparrow n \leq p\left\lceil n\right.$ in $\mathbb{R}^{n}$ for all $n$. Let $q \leq^{*} p$ if for all $n, q\left\lceil n \Vdash q(n) \leq^{*} p(n)\right.$.

For each $n<\omega, \mathbb{R}_{\omega}$ factors as $\mathbb{R}^{n} * \mathbb{R}_{n}^{\omega}$ in the obvious way, where $\mathbb{R}_{n}^{\omega}$ is a name for the iteration defined using the sequence $\left\langle\mathbb{R}_{m}: n \leq m<\omega\right\rangle$ in the generic extension by $\mathbb{R}^{n}$.

Proposition 8.5. The poset $\mathbb{R}^{\omega}$ satisfies the Prikry property.

Proof. Let $\varphi$ be a statement in the forcing language for $\mathbb{R}^{\omega}$ and let $p$ be a condition in $\mathbb{R}^{\omega}$. Suppose for a contradiction that there is no direct extension of $p$ which decides $\varphi$. We inductively define a $\leq^{*}$-decreasing sequence $\left\langle q_{n}: n\langle\omega\rangle\right.$ in $\mathbb{R}^{\omega}$ as follows. Let $q_{0}=p$. Suppose $q_{n}$ is defined for some $n \geq 0$. Let $q_{n+1} \uparrow[n+1, \omega)$ be a sequence of names such that $q_{n} \uparrow(n+1)$ forces the following: if there is a direct extension of $q_{n} \uparrow[n+1, \omega)$ which decides $\varphi$, then $q_{n+1} \uparrow[n+1, \omega)$ is such a direct extension; otherwise $q_{n+1} \uparrow[n+1, \omega)=q_{n} \uparrow[n+1, \omega)$. Define $q_{n+1} \uparrow n=q_{n} \uparrow n$. Let $q_{n+1}(n)$ denote a name for a direct extension of $q_{n}(n)$ which decides whether $q_{n+1} \uparrow[n+1, \omega)$ decides $\varphi$, and if so, which way it decides $\varphi$.

Now define $q=\left\langle q_{(n+1)}(n): n<\omega\right\rangle$. Clearly $q \leq^{*} p$. Also note that for all $n<\omega$, $q \uparrow n=q_{n} \uparrow n$. By assumption there is no direct extension of $q$ which decides $\varphi$. For each $r \leq q$ let $b_{r}$ be the set of $m<\omega$ such that $r \uparrow m$ does not force that $r(m) \leq^{*} q(m)$. Then $b_{r} \subseteq a_{r}$, so $b_{r}$ is finite. Choose $r \leq q$ which decides $\varphi$ such that $\left|b_{r}\right|$ is minimal. Let $n=\max \left(b_{r}\right)$, which exists since $r$ is not a direct extension of $q$. Fix $l<2$ such that $r$ forces ${ }^{l} \varphi$. Then

$$
r \uparrow n \Vdash r(n) \Vdash\left(r \uparrow[n+1, \omega) \leq^{*} q_{n} \uparrow[n+1, \omega)\right) \&\left(r \uparrow[n+1, \omega) \Vdash^{l} \varphi\right) .
$$


Since $r\left\lceil n \leq q_{n}\left\lceil n\right.\right.$ and $q\left\lceil[n+1, \omega) \leq^{*} q_{n+1}\lceil[n+1, \omega)\right.$, by the definition of $q(n)$ we have

$$
r\left\lceil n \Vdash q(n) \Vdash q \uparrow[n+1, \omega) \Vdash{ }^{l} \varphi .\right.
$$

It follows that $s=\left(r\lceil n)^{\wedge} q(n)^{\wedge}(q \uparrow[n+1, \omega))\right.$ forces ${ }^{l} \varphi$. But $b_{s}=b_{r} \backslash\{n\}$, which contradicts the minimality of $\left|b_{r}\right|$.

Now we analyze preservation of cardinals and changes in cofinality. We need the following simple lemma.

Lemma 8.6. Suppose $\xi$ is less than $\kappa$ and $p$ is a condition in $\mathbb{R}^{\omega}$. Assume that for each $n<\omega, p\left\lceil n\right.$ forces that if $a$ is in $P_{\kappa} \kappa_{n}$ and appears in $p(n)$, and the order of $a \cap \kappa$ is greater than 0 , then $a \cap \kappa>\xi$. If $\left\{p_{i}: i<\xi\right\}$ is a collection of direct extensions of $p$, then there is $q$ such that $q \leq^{*} p_{i}$ for $i<\xi$.

Proof. Define $q$ inductively. Suppose $q \uparrow n$ is defined so that $q\left\lceil n \leq^{*} p_{i} \uparrow n\right.$ for $i<\xi$. Apply Lemma 3.1 to find a name $q(n)$ such that $q\lceil n$ forces that $q(n) \leq^{*} p_{i}(n)$ for $i<\xi$.

Let $G$ be generic for $\mathbb{R}^{\omega}$ over $V$. For $n<\omega$ let $G\lceil n$ denote the set $\{p\lceil n: p \in$ $G\}$. Then $G\left\lceil n\right.$ is generic for $\mathbb{R}^{n}$ over $V$.

Lemma 8.7. Suppose that $\mu<\kappa$. Then there is $m<\omega$ such that $\mathcal{P}^{V[G]}(\mu)$ is in $V[G \uparrow m]$.

Proof. Suppose that $p$ is a condition in $G$. Let $n=\max \left(a_{p}\right)$. For each $m>n$ write $p(m)=\left\langle\dot{A}_{m}\right\rangle$, where $\dot{A}_{m}$ is a name for a subset of $P_{\kappa} \kappa_{m}$. Define $q$ by letting $q \uparrow(n+1)=p\left\lceil(n+1)\right.$ and for $m>n$, let $q(m)$ be a name for $\left\langle\left\{a \in \dot{A}_{m}: a \cap \kappa>\right.\right.$ $\mu\}\rangle$.

We claim that $q$ forces that $\mathcal{P}(\mu)$ is in $V[G \uparrow(n+1)]$. Suppose that $r \leq q$ and $r$ forces $\dot{f}: \mu \rightarrow 2$. For each $i<\mu$ let $s_{i}$ be a name for a condition in $\mathbb{R}_{n+1}^{\omega}$ such that $r \uparrow(n+1)$ forces that $s_{i} \leq^{*} r \uparrow[n+1, \omega)$ and $s_{i}$ decides the statement $\dot{f}(i)=0$. Apply Lemma 8.6 to $\mathbb{R}_{n+1}^{\omega}$ to find a name $s$ such that $r \uparrow(n+1)$ forces that $s \leq^{*} s_{i}$ for $i<\mu$. Then $r \uparrow(n+1)$ forces that $s$ decides all the values $\dot{f}(i)$ for $i<\mu$. Therefore $(r \uparrow(n+1))^{\wedge} s$ is a refinement of $r$ which forces that $\dot{f}$ is in $V[G \uparrow(n+1)]$.

In particular, if $\mu<\kappa$ is a cardinal in $V$ which is collapsed in $V[G]$, then there is $m<\omega$ such that $\mu$ is collapsed in $V[G\lceil m]$. Also if $\mu<\kappa$ and $\operatorname{cf}(\mu)=\delta$ in $V[G]$, then there is $m<\omega$ such that $\operatorname{cf}(\mu)=\delta$ in $V[G\lceil m]$.

Proposition 8.8. In $V[G], \kappa$ is a strong limit singular cardinal with cofinality $\omega$.

Proof. For each $n$ let $C_{n}$ be the Radin club on $P_{\kappa} \kappa_{n}$ in $V[G]$. Let $D=\left\{\min \left(C_{n}\right) \cap \kappa\right.$ : $n<\omega\}$. We claim that $D$ is unbounded in $\kappa$, and therefore $\operatorname{cf}(\kappa)=\omega$ in $V[G]$. Fix $\xi<\kappa$. Suppose that $p$ is in $G$ and let $n=\max \left(a_{p}\right)$. For each $m>n$ write $p(m)=\left\langle\dot{A}_{m}\right\rangle$. Define $q \leq p$ by letting $q \uparrow(n+1)=p\lceil(n+1)$, and for $m>n$ let $q(m)$ be a name for $\left\langle\left\{a \in \dot{A}_{m}: a \cap \kappa>\xi\right\}\right\rangle$. Then $q$ forces that for all $m>n$, $\min \left(C_{m}\right) \cap \kappa>\xi$. So $D$ is unbounded in $\kappa$.

To show that $\kappa$ is a strong limit cardinal in $V[G]$ it suffices to show that for all $\mu<\kappa, 2^{\mu}<\kappa$. Fix $\mu<\kappa$. By Lemma 8.7 there is $m<\omega$ such that $\mathcal{P}^{V[G]}(\mu)=$ $\mathcal{P}^{V[G\lceil m]}(\mu)$. But $\kappa$ is supercompact in $V[G\lceil m]$. 
Suppose we are in the case that $\kappa_{n}<\kappa_{n+1}$ for $n<\omega$. Let $\kappa_{\omega}=\sup \left(\left\{\kappa_{n}: n<\right.\right.$ $\omega\})$.

Proposition 8.9. Suppose that $\kappa \leq \mu<\kappa_{\omega}$ and $\operatorname{cf}(\mu) \geq \kappa$ in $V$. Then $\operatorname{cf}(\mu)=\omega$ in $V[G]$. Therefore all cardinals in the interval $\left(\kappa, \kappa_{\omega}\right]$ are collapsed to $\kappa$.

Proof. Let $C_{n}$ be the Radin club on $P_{\kappa} \kappa_{n}$ in $V[G]$. Define $D_{\mu}=\left\{\sup \left(\min \left(C_{n}\right) \cap \mu\right)\right.$ : $n<\omega\}$. As in the proof of Proposition 8.8, $D_{\mu}$ is an unbounded subset of $\mu$.

Proposition 8.10. The poset $\mathbb{R}^{\omega}$ is $\kappa_{\omega}^{+}$-c.c.

Proof. Suppose for a contradiction that $A$ is an antichain in $\mathbb{R}^{\omega}$ with size $\kappa_{\omega}^{+}$. Then there is $B \subseteq A$ with size $\kappa_{\omega}^{+}$and a finite set $a \subseteq \omega$ such that for all $p$ in $B, a_{p}=a$. Let $n=\max (a)$. We claim that the set $\left\{p\lceil(n+1): p \in B\}\right.$ is an antichain in $\mathbb{R}^{n+1}$, which will contradict the fact that $\mathbb{R}^{n+1}$ is $\left(\kappa_{n}^{<\kappa}\right)^{+}$-c.c. Suppose that $p$ and $q$ are in $B$ and $r$ is below both $p\lceil(n+1)$ and $q\lceil(n+1)$. Then $r$ forces that $p\lceil[n+1, \omega)$ and $q \uparrow[n+1, \omega)$ are both direct extensions of 1 and hence are compatible. Let $u$ be a name such that $r$ forces that $u$ is below both $q\lceil[n+1, \omega)$ and $p\lceil[n+1, \omega)$. Then $r^{\wedge} u$ is below both $p$ and $q$, which is impossible.

Let us summarize the situation. In $V[G], \kappa$ is a strong limit singular cardinal with cofinality $\omega$. If $\mu$ is a cardinal in $V$ such that $\kappa<\mu \leq \kappa_{\omega}$, then $\mu$ is collapsed to $\kappa$ in $V[G]$. The successor of $\kappa_{\omega}$ in $V$ is preserved and becomes the successor of $\kappa$ in $V[G]$.

\section{ITERATING RADIN FORCING OVER DIFFERENT CARDINALS}

Magidor [6] introduced a method for iterating Prikry forcing over different cardinals. In this section we show that the same method works more generally for Radin forcing.

First we show how to iterate Radin forcing using coherent sequences of arbitrary length. At each stage we use a coherent sequence derived from a supercompact ultrafilter. This works as we will see because the iteration preserves supercompactness. Afterwards we refine this construction, iterating Radin forcing defined from coherent sequences without appealing to a larger supercompact ultrafilter.

Suppose that $A$ is a set of cardinals satisfying the following properties:

(1) There is a map $\alpha \mapsto \lambda_{\alpha}$ defined on $A$, and every $\beta$ in $A$ is closed under this map.

Let $A^{*}$ denote the set $\bigcup\left\{\left[\alpha, \lambda_{\alpha}^{+}\right]: \alpha \in A\right\}$.

(2) For each $\alpha$ in $A$, there is a normal ultrafilter $U_{\alpha}$ on $P_{\alpha}\left(2^{2^{\left(\lambda_{\alpha}^{<\alpha}\right)}}\right)$ with elementary embedding $j_{\alpha}: V \rightarrow \operatorname{Ult}\left(V, U_{\alpha}\right)$ such that $\min \left(j\left(A^{*}\right) \backslash \alpha\right)>\lambda_{\alpha}$. In particular, $U_{\alpha}$ concentrates on $\alpha \backslash A^{*}$.

We would like to add a Radin club to $P_{\alpha} \lambda_{\alpha}$ for each $\alpha$ in $A$.

Define by recursion an iterated forcing $\left\langle\mathbb{M}_{\alpha}, \mathbb{R}_{\alpha}: \alpha \leq \sup (A)\right\rangle$, maintaining the following recursion hypotheses:

\section{Recursion Hypotheses:}

(1) If $\alpha$ is a strong limit cardinal closed under the map $\beta \mapsto \lambda_{\beta}$, then $\left|\mathbb{M}_{\alpha}\right| \leq 2^{\alpha}$ and $\mathbb{M}_{\alpha}$ is $\alpha^{+}$-c.c.

Suppose that $\alpha$ is in $A$.

(2) The poset $\mathbb{M}_{\alpha}$ forces there is a normal ultrafilter $U_{\alpha}^{*}$ which extends $U_{\alpha}$.

(3) The cardinals $\lambda_{\alpha}^{<\alpha}, 2^{\left(\lambda_{\alpha}^{<\alpha}\right)}$, and $2^{2^{\left(\lambda_{\alpha}^{<\alpha}\right)}}$ are computed the same in both $V$ 
and any generic extension by $\mathbb{M}_{\alpha}$.

(4) $\left|\mathbb{M}_{\alpha+1}\right| \leq 2^{\left(\lambda_{\alpha}^{<\alpha}\right)}$.

Let $\alpha \leq \sup (A)$ and assume that $\mathbb{M}_{\alpha}$ is defined and satisfies the recursion hypotheses. If $\alpha$ is not in $A$ then let $\mathbb{R}_{\alpha}$ be a name for the trivial poset and define $\mathbb{M}_{\alpha+1}=\mathbb{M}_{\alpha} * \mathbb{R}_{\alpha}$.

Suppose that $\alpha$ is in $A$. Let $G_{\alpha}$ be generic for $\mathbb{M}_{\alpha}$ over $V$. By the recursion hypotheses there is a normal ultrafilter $U_{\alpha}^{*}$ in $V\left[G_{\alpha}\right]$ which extends $U_{\alpha}$. By Lemma 1.5 and Proposition 2.2, for each $\xi \leq\left(2^{\left(\lambda_{\alpha}^{<\alpha}\right)}\right)^{+}$there is a coherent sequence of ultrafilters $\vec{U}=\left\langle U(\beta, i): \beta \leq \alpha, i<o^{\vec{U}}(\beta)\right\rangle$ on $P_{\alpha} \lambda_{\alpha}$ such that each $U(\beta, i)$ concentrates on $\beta \backslash A^{*}$, the domain of $o^{\vec{U}}$ is disjoint from $A^{*} \cap \alpha$, and $o^{\vec{U}}(\kappa)=\xi$. Choose some such coherent sequence on $P_{\alpha} \lambda_{\alpha}$ and let $\mathbb{R}_{\alpha}$ be the Radin forcing defined from this coherent sequence. Let $\mathbb{M}_{\alpha+1}=\mathbb{M}_{\alpha} * \mathbb{R}_{\alpha}$. The poset $\mathbb{M}_{\alpha}$ is $\alpha^{+}$c.c., has size no larger than $2^{\alpha}$, and forces that $\left|\mathbb{R}_{\alpha}\right| \leq 2^{\left(\lambda_{\alpha}^{<\alpha}\right)}$. So $\left|\mathbb{M}_{\alpha+1}\right| \leq 2^{\left(\lambda_{\alpha}^{<\alpha}\right)}$.

Suppose that $\alpha$ is a limit ordinal and $\mathbb{M}_{\beta}$ is defined for $\beta<\alpha$. Define $\mathbb{M}_{\alpha}$ as the set of functions $p$ with domain $\alpha$ such that for all $\beta<\alpha, p\left\lceil\beta\right.$ is in $\mathbb{M}_{\beta}$; moreover, there exists a finite set $a_{p} \subseteq \alpha$ such that for $\beta<\alpha, \beta$ is in $\alpha \backslash a_{p}$ iff $p\lceil\beta$ forces that $p(\beta)$ is a direct extension 1 in $\mathbb{R}_{\beta}$. Let $q \leq p$ if $q\lceil\beta \leq p\lceil\beta$ for $\beta<\alpha$. Let $q \leq^{*} p$ if for all $\beta<\alpha, q\left\lceil\beta\right.$ forces that $q(\beta) \leq{ }^{*} p(\beta)$.

The iteration $\mathbb{M}_{\alpha}$ satisfies the Prikry property; for a proof see [2] or [6].

We verify the recursion hypotheses. Suppose that $\alpha$ is a strong limit cardinal closed under the map $\beta \mapsto \lambda_{\beta}$. First assume $\sup (A)=\gamma<\alpha$. Then $\gamma$ is a strong limit cardinal closed under the map $\beta \mapsto \lambda_{\beta}$, so by recursion $\left|\mathbb{M}_{\alpha}\right|=\left|\mathbb{M}_{\gamma+1}\right|<\alpha$. Otherwise $\sup (A)=\alpha$. Then for each $\gamma<\alpha,\left|\mathbb{M}_{\gamma}\right|<\alpha$. So $\bigcup\left\{\mathbb{M}_{\gamma}: \gamma<\alpha\right\}$ has size $\alpha$, and therefore $\mathbb{M}_{\alpha}$ has size at most $2^{\alpha}$.

Suppose that $B \subseteq \mathbb{M}_{\alpha}$ with $|B|=\alpha^{+}$. Since there are only $\alpha$ many finite subsets of $\alpha$, there is a finite set $a$ and $C \subseteq B$ with size $\alpha^{+}$such that $a_{p}=a$ for all $p$ in $C$. But there are fewer than $\alpha$ many possibilities for $p\left\lceil a_{p}\right.$. Let $p$ and $q$ be in $C$ such that $p\lceil a=q\lceil a$. Then $p$ and $q$ are compatible.

Lemma 9.1. Suppose that $p$ is a condition in $\mathbb{M}_{\alpha}, \xi<\min (A)$, and at least one of the following two statements holds:

(1) $p$ is a direct extension of 1 ,

(2) for all $\beta$ in $A \cap \alpha, p\left\lceil\beta\right.$ forces that any a in $P_{\beta} \lambda_{\beta}$ appearing in $p(\beta)$ satisfies that $a \cap \beta>\xi$.

If $\left\{p_{i}: i<\xi\right\}$ is a family of direct extensions of $p$, there is $q$ such that $q \leq^{*} p_{i}$ for $i<\xi$.

Proof. For (1), write $p_{i}(\beta)=\left\langle\dot{A}_{i}(\beta)\right\rangle$ for $\beta$ in $A \cap \alpha$, where $\dot{A}_{i}(\beta)$ is a name for a subset of $P_{\beta} \lambda_{\beta}$. Define $q$ by letting $q(\beta)=\left\langle\bigcap\left\{\dot{A}_{i}(\beta): i<\xi\right\}\right\rangle$. Since $\xi<\min (A)$, the completeness of the ultrafilters implies that $q$ is a condition in $\mathbb{M}_{\alpha}$. The proof of (2) is similar, using Lemma 3.1.

Lemma 9.2. Suppose that $p$ is a condition in $\mathbb{M}_{\alpha}$ and $\xi<\min (A)$. Assume that for all $\beta$ in $A \cap \alpha, p\left\lceil\beta\right.$ forces that whenever a in $P_{\beta} \lambda_{\beta}$ is either in the finite sequence of $p(\beta)$ or in a measure one set appearing in $p(\beta)$, then $a \cap \beta>\xi$. Then $p$ forces that no new subsets of $\xi$ are added. 
Proof. Any $q \leq p$ satisfies (2) of Lemma 9.1. Suppose that $q \leq p$ and $q$ forces $\dot{f}: \xi \rightarrow 2$. For each $i<\xi$ let $q_{i} \leq^{*} q$ decide the value of $\dot{f}(i)$. Then there is $r$ such that $r \leq^{*} q_{i}$ for $i<\xi$. Clearly $r$ forces that $\dot{f}$ is in the ground model.

Now we verify the second recursion hypothesis.

Proposition 9.3. Suppose that $\alpha$ is in $A$. Then the poset $\mathbb{M}_{\alpha}$ forces there is a normal ultrafilter $U_{\alpha}^{*}$ which extends $U_{\alpha}$.

Proof. If $\alpha$ is not a limit point of $A$, then $\left|\mathbb{M}_{\alpha}\right|<\alpha$, so $U_{\alpha}$ can be lifted. Suppose that $\alpha$ is a limit point of $A$.

Write $j: V \rightarrow M=\operatorname{Ult}\left(V, U_{\alpha}\right)$. Let $\beta=\min (j(A) \backslash \alpha)$, which by assumption is larger than $\lambda_{\alpha}$. Factor $j\left(\mathbb{M}_{\alpha}\right)=\mathbb{M}_{\alpha} * \mathbb{M}_{\beta, j(\alpha)}$. Write $\mu_{\alpha}=2^{2^{\left(\lambda_{\alpha}^{<\alpha}\right)}}$. Note that $\beta>\mu_{\alpha}$.

Let $G_{\alpha}$ be generic for $\mathbb{M}_{\alpha}$ over $V$. Define $U_{\alpha}^{*}$ in $V\left[G_{\alpha}\right]$ by letting $X$ be in $U_{\alpha}^{*}$ iff $X \subseteq P_{\alpha} \mu_{\alpha}$ and there is a name $\dot{X}$ for $X$ and $s \leq^{*} 1$ in $\mathbb{M}_{\beta, j(\beta)}$ such that $s$ forces over $M\left[G_{\alpha}\right]$ that $j$ " $\mu_{\alpha} \in j(\dot{X})$.

We show that the definition does not depend on the choice of $\dot{X}$. Suppose that $X=\dot{X}^{G_{\alpha}}=\dot{Y}^{G_{\alpha}}$. By the Prikry property for $\mathbb{M}_{\beta, j(\alpha)}$, there is $s_{\dot{X}}$ (respectively $s_{\dot{Y}}$ ) directly extending 1 which decides whether $j$ " $\mu_{\alpha}$ is in $j(\dot{X})$ (respectively, in $j(\dot{Y}))$. Fix $p$ in $G_{\alpha}$ which forces that $\dot{X}=\dot{Y}$. Then $j\left(a_{p}\right)=a_{p}$, so $j(p)=p^{\wedge} r$ where $r$ is a direct extension of 1 in $\mathbb{M}_{\beta, j(\alpha)}$. Moreover, $r$ forces over $M\left[G_{\alpha}\right]$ that $j(\dot{X})=j(\dot{Y})$. Since $r, s_{\dot{X}}$, and $s_{\dot{Y}}$ are direct extensions of 1 , they are compatible. So $s_{\dot{X}}$ and $s_{\dot{Y}}$ must decide their respective statements the same way.

Similar arguments show that $U_{\alpha}^{*}$ is an ultrafilter which extends $U_{\alpha}$. We prove that $U_{\alpha}^{*}$ is normal. Suppose that $f: P_{\alpha} \mu_{\alpha} \rightarrow \mu_{\alpha}$ is regressive. Let $\dot{f}$ be a name for $f$ and fix $p$ in $G_{\alpha}$ which forces that $\dot{f}$ is regressive. Then $j(p)=\widehat{ } \widehat{ } r$, where $r$ is a direct extension of 1 and $r$ forces over $M\left[G_{\alpha}\right]$ that $j(\dot{f})$ is regressive. For each $i<\mu_{\alpha}$ choose $s_{i} \leq^{*} 1$ in $\mathbb{M}_{\beta, j(\alpha)}$ which decides the statement $j(\dot{f})\left(j " \mu_{\alpha}\right)=j(i)$. By Lemma 9.1 there is $s$ such that $s \leq^{*} s_{i}$ for all $i$. Clearly there must exist $i$ such that $s$ forces $j(\dot{f})\left(j\right.$ " $\left.\mu_{\alpha}\right)=j(i)$. But then $\left\{a \in P_{\alpha} \mu_{\alpha}: f(a)=i\right\}$ is in $U_{\alpha}^{*}$.

The proof of the third recursion hypothesis is similar to the proof of Lemma 8.4. This completes the construction of the iteration.

Suppose that $\alpha$ is in $A$ and $\alpha^{\prime}$ is the least element of $A$ greater than $\alpha$. Write $\mathbb{M}_{\sup (A)}=\mathbb{M}_{\alpha+1} * \mathbb{M}_{\text {tail }}$. Unlike the case of an Easton support iteration of strategically closed posets or even a Magidor iteration of Prikry forcing, the poset $\mathbb{M}_{\text {tail }}$ will add bounded subsets to $\alpha^{\prime}$ over the generic extension by $\mathbb{M}_{\alpha+1}$. One might worry that this tail iteration will damage the universe below $\alpha^{\prime}$ in such a way that it ruins the effect achieved in forcing with $\mathbb{M}_{\alpha+1}$. It turns out this is not true, due to a nice factorization of the tail iteration.

Theorem 9.4. Suppose that $\alpha$ is in $A$ and $\alpha^{\prime}=\min (A \backslash(\alpha+1))$. Write $\mathbb{M}_{\sup (A)}=$ $\mathbb{M}_{\alpha+1} * \mathbb{M}_{\text {tail }}$. Let $G_{\alpha+1}$ be generic for $\mathbb{M}_{\alpha+1}$ over $V$. Then in $V\left[G_{\alpha+1}\right]$, there is a dense set of $q$ in $\mathbb{M}_{\text {tail }}$ such that $\mathbb{M}_{\text {tail }} / q$ factors into a finite iteration

$$
\mathbb{P}_{0} * \cdots * \mathbb{P}_{n}
$$

such that for each $i \leq n$, either $\mathbb{P}_{i}$ does not add subsets to $\lambda_{\alpha}^{+}$or $\mathbb{P}_{i}$ is a Radin forcing on some $P_{\gamma} \gamma^{\prime}$ where $\gamma^{\prime}<\alpha$. 
Proof. Working in $V\left[G_{\alpha+1}\right]$, let $p$ be in $\mathbb{M}_{\text {tail }}$. We define $q \leq p$ as follows. Fix $\xi$ in $A \backslash(\alpha+1)$. Let $\vec{U}$ be a name for the coherent sequence such that $\mathbb{R}_{\xi}=\mathbb{R}(\vec{U})$. Then $p\left\lceil\xi\right.$ forces that for all $a$ in $P_{\xi} \lambda_{\xi}$ appearing in the finite sequence of $p(\xi)$, either $a \cap \xi<\alpha$ or $a \cap \xi>\lambda_{\alpha}^{+}$. This is true since $\alpha$ is in $A$ and therefore the interval $\left[\alpha, \lambda_{\alpha}^{+}\right]$is disjoint from the domain of $o^{\vec{U}}$. Write $p(\xi)=\left\langle\vec{d}_{\xi}, \dot{A}_{\xi}\right\rangle$. Define $q(\xi)=\left\langle\vec{d}_{\xi}, \dot{B}_{\xi}\right\rangle$, where $\dot{B}_{\xi}=\left\{a \in \dot{A}_{\xi}: a \cap \xi>\lambda_{\alpha}^{+}\right\}$.

Write $a_{q} \backslash\left\{\alpha^{\prime}, \sup (A)\right\}=\left\{\xi_{1}, \ldots, \xi_{n-1}\right\}$, and let $\xi_{0}=\alpha^{\prime}$ and $\xi_{n}=\sup (A)$. For $i \leq n$ write $s_{i}=q \uparrow\left(\xi_{i}, \xi_{i+1}\right)$. Then $\mathbb{M}_{\text {tail }} / q$ factors as

$\left(\mathbb{R}_{\xi_{0}} / q\left(\xi_{0}\right)\right) *\left(\mathbb{M}_{\left(\xi_{0}, \xi_{1}\right)} / s_{0}\right) * \ldots *\left(\mathbb{R}_{\xi_{n-1}} / q\left(\xi_{n-1}\right)\right) *\left(\mathbb{M}_{\left(\xi_{n-1}, \xi_{n}\right)} / s_{n-1}\right) *\left(\mathbb{R}_{\xi_{n}} / q\left(\xi_{n}\right)\right)$.

By Lemma 9.2 and the definition of $q$, each $\mathbb{M}_{\left(\xi_{i}, \xi_{i+1}\right)} / s_{i}$ does not add any subsets to $\lambda_{\alpha}^{+}$. By the definition of $q$, the Factorization Lemma, and Lemma 5.8(3), each $\mathbb{R}_{\xi_{i}} / q\left(\xi_{i}\right)$ factors into a product of a Radin forcing below $\alpha$ by a Radin forcing which adds no subsets of $\lambda_{\alpha}^{+}$.

We show now that the stationarity of $A$ is always destroyed. Let $\alpha$ be a limit point of $A$ with cofinality greater than $\omega$. Suppose that $G_{\alpha}$ is generic for $\mathbb{M}_{\alpha}$ over $V$. For each $\xi$ in $A \cap \alpha$ let $c_{\xi}$ denote the Radin club added by a generic for the Radin forcing on $P_{\xi} \lambda_{\xi}$.

Proposition 9.5. Define $C_{\mathbb{M}_{\alpha}}$ in $V\left[G_{\alpha}\right]$ as the set of $\beta<\alpha$ such that for all $\xi \geq \beta$ in $A \cap \alpha, \min \left(c_{\xi}\right) \cap \xi \geq \beta$. Then $C_{\mathbb{M}_{\alpha}}$ is a club subset of $\alpha$ disjoint from $A \cap \alpha$.

Proof. If $\xi$ is in $A \cap \alpha$ then $\min \left(c_{\xi}\right) \cap \xi<\xi$, so $\xi$ is not in $C_{\mathbb{M}_{\alpha}}$. Suppose that $C$ is a bounded subset of $C_{\mathbb{M}_{\alpha}}$ and let $\beta=\sup (C)$. We show that $\beta$ is in $C_{\mathbb{M}_{\alpha}}$. Consider $\xi \geq \beta$ in $A \cap \alpha$. For all $\beta^{\prime}$ in $C, \min \left(c_{\xi}\right) \cap \xi \geq \beta^{\prime}$. Therefore $\min \left(c_{\xi}\right) \cap \xi \geq \sup (C)=\beta$.

To show that $C_{\mathbb{M}_{\alpha}}$ is unbounded in $\alpha$, fix $\gamma<\alpha$ and let $p$ be in $G_{\alpha}$. Fix an ordinal $\beta<\alpha$ not in $A \cap \alpha$ such that $\beta$ is larger than $\max \left(a_{p}\right)$ and $\gamma$. Define $q$ as follows. Let $q \uparrow(\beta+1)=p\lceil(\beta+1)$. Fix $\beta<\xi<\alpha$. If $\xi$ is not in $A \cap \alpha$ then let $q(\xi)=p(\xi)$. Otherwise write $p(\xi)=\left\langle\dot{A}_{\xi}\right\rangle$. Let $q(\xi)$ be a name for the condition $\left\langle\left\{a \in \dot{A}_{\xi}: a \cap \xi>\beta\right\}\right\rangle$. Then $q \leq p$ and $q$ forces that $\beta$ is in $C_{\mathbb{M}_{\alpha}}$.

Now we will refine this construction somewhat. Ideally we would like to do something like the following. Start with a set of cardinals $A$ and a coherent sequence of ultrafilters on each $\alpha$ in $A$. Define the Magidor iteration as above. Maintain the recursion hypothesis that for each $\alpha$ in $A$, the coherent sequence on $\alpha$ can be lifted after forcing with $\mathbb{M}_{\alpha}$. This idea can be made to work when the coherent sequences are short using Proposition 2.1. But we do not know whether a long coherent sequence on $\alpha$ can be lifted after forcing with $\mathbb{M}_{\alpha}$ without making further assumptions.

Suppose that $A$ is a set of cardinals satisfying the following properties:

(1) For each $\alpha$ in $A$ there is a $\triangleleft$-increasing sequence $\left\langle U_{i}^{\alpha}: i<\beta_{\alpha}\right\rangle$ of normal ultrafilters on $\alpha$, for some $\beta_{\alpha} \leq \alpha$.

(3) Each $U_{i}^{\alpha}$ contains $\alpha \backslash A$.

Note that the hypotheses of Proposition 2.1 are satisfied.

Define by recursion an iterated forcing $\left\langle\mathbb{M}_{\alpha}, \mathbb{R}_{\alpha}: \alpha \leq \sup (A)\right\rangle$ using the same definition as above. The only difference is that we need to prove for all $\alpha$ in $A$, $\mathbb{M}_{\alpha}$ forces that the sequence $\left\langle U_{i}^{\alpha}: i<\beta_{\alpha}\right\rangle$ can be lifted in such a way that it still satisfies the hypotheses of Proposition 2.1. Clearly it suffices to prove that the lifted sequence is $\triangleleft$-increasing. Then we apply Proposition 2.1 to obtain a coherent 
sequence $\vec{U}=\left\langle U(\beta, i): \beta \leq \alpha, i<o^{\vec{U}}(\beta)\right\rangle$ on $\alpha$ in the generic extension by $\mathbb{M}_{\alpha}$ such that $o^{\vec{U}}(\alpha)=\beta_{\alpha}$, each $U(\beta, i)$ contains $\beta \backslash A$, and the domain of $o^{\vec{U}}$ is disjoint from $A \cap \alpha$. Let $\mathbb{R}_{\alpha}$ be the Radin forcing defined from this coherent sequence.

To prove that the sequence on $\alpha$ can be lifted, we need a more complicated recursion hypothesis. In addition to recursion hypotheses 1, 3, and 4 in the previous construction, we maintain the following.

Recursion Hypothesis: Suppose that $\alpha \leq \sup (A)$ is a limit point of $A$. Assume $U$ is a normal ultrafilter on $\alpha$ which contains $\alpha \backslash A$. Write $j_{U}\left(\mathbb{M}_{\alpha}\right)=\mathbb{M}_{\alpha} * \mathbb{M}_{\alpha, j_{U}(\alpha)}$. Let $G_{\alpha}$ be generic for $\mathbb{M}_{\alpha}$ over $V$. Define $U^{*}$ in $V\left[G_{\alpha}\right]$ by letting $X$ be in $U^{*}$ iff $X \subseteq \alpha$, there is a name $\dot{X}$ for $X$, and there is $s \leq^{*} 1$ in $\mathbb{M}_{\alpha, j_{U}(\alpha)}$ such that $s$ forces over $M_{U}\left[G_{\alpha}\right]$ that $\alpha \in j_{U}(\dot{X})$. Then $U^{*}$ is a normal ultrafilter on $\alpha$ which extends $U$. Moreover, if $U \triangleleft U_{1}$ and $j_{1}: V \rightarrow M_{1}=\operatorname{Ult}\left(V, U_{1}\right)$, then $U^{*}$ is in $M_{1}\left[G_{\alpha}\right]$ and satisfies the same definition in $M_{1}\left[G_{\alpha}\right]$ as it does in $V\left[G_{\alpha}\right]$.

Suppose that $\mathbb{M}_{\alpha}$ is defined and $\alpha$ is a limit point of $A$. We verify the recursion hypothesis. Let $U$ be a normal ultrafilter on $\alpha$ which contains $\alpha \backslash A$. Define $U^{*}$ as in the statement of the recursion hypothesis. The proof that $U^{*}$ is a normal ultrafilter on $\alpha$ is similar to the proof of Proposition 9.3. Suppose that $U \triangleleft U_{1}$ and $j_{1}: V \rightarrow M_{1}=\operatorname{Ult}\left(V, U_{1}\right)$. Write $k: M_{1} \rightarrow N=\operatorname{Ult}\left(M_{1}, U\right)$. Then $j_{U}\left\lceil M_{1}=k\right.$ by Lemma 1.4(2), so $k\left(\mathbb{M}_{\alpha}\right)=\mathbb{M}_{\alpha} * \mathbb{M}_{\alpha, j_{U}(\alpha)}$. Define $U^{* *}$ extending $U$ in $M_{1}\left[G_{\alpha}\right]$ exactly as $U^{*}$ was defined in $V\left[G_{\alpha}\right]$, using $k$ in place of $j_{U}$. Since $\mathbb{M}_{\alpha}$ is $\alpha^{+}$-c.c., $M_{1}\left[G_{\alpha}\right]$ is $\alpha$-closed in $V\left[G_{\alpha}\right]$. So $U^{* *}$ really is an ultrafilter. We show that $U^{* *}$ and $U^{*}$ are equal by proving $U^{* *} \subseteq U^{*}$. Suppose $\dot{X}$ is a name in $M_{1}\left[G_{\alpha}\right], s$ is a direct extension of 1 in $\mathbb{M}_{\alpha, j_{U}(\alpha)}$, and $s$ forces over $N\left[G_{\alpha}\right]$ that $\alpha \in k(\dot{X})$. Then $s$ forces over $N\left[G_{\alpha}\right]$ that $\alpha \in j_{U}(\dot{X})$. Since $N\left[G_{\alpha}\right] \subseteq M_{U}\left[G_{\alpha}\right]$ by Lemma 1.4(1) and the fact that the statement $\alpha \in j_{U}(\dot{X})$ is upwards absolute, $s$ forces over $M\left[G_{\alpha}\right]$ that $\alpha \in j_{U}(\dot{X})$. So $\dot{X}^{G_{\alpha}}$ is in $U^{*}$.

This completes the proof of the main recursion hypothesis. We omit the proofs of the other recursion hypotheses since they are the same as above.

Now we prove that when $\alpha$ is in $A, \mathbb{M}_{\alpha}$ forces that the sequence of ultrafilters on $\alpha$ can be lifted as desired. Suppose that $\alpha$ is in $A$ and $\mathbb{M}_{\alpha}$ is defined. Let $G_{\alpha}$ be generic for $\mathbb{M}_{\alpha}$ over $V$.

First assume that $\alpha$ is not a limit point of $A$. Then $\left|\mathbb{M}_{\alpha}\right|<\alpha$. So each $U_{i}^{\alpha}$ generates a normal ultrafilter $\left(U_{i}^{\alpha}\right)^{*}$ in the generic extension. We prove that $\left\langle\left(U_{i}^{\alpha}\right)^{*}: i<\beta_{\alpha}\right\rangle$ is $\triangleleft$-increasing.

Fix $\alpha_{0}<\alpha_{1}<\beta_{\alpha}$. We define $g_{\alpha_{0}}^{\alpha_{1}}$ as follows. The domain of $g_{\alpha_{0}}^{\alpha_{1}}$ is the set of $\xi<\alpha$ larger than $\left|\mathbb{M}_{\alpha}\right|$ such that $f_{\alpha_{0}}^{\alpha_{1}}(\xi)$ is a normal ultrafilter in $V$ on $\xi$. Let $g_{\alpha_{0}}^{\alpha_{1}}(\xi)$ denote the ultrafilter generated by $f_{\alpha_{0}}^{\alpha_{1}}(\xi)$ in $V\left[G_{\alpha}\right]$.

Write $j_{\alpha_{1}}^{*}: V\left[G_{\alpha}\right] \rightarrow M_{\alpha_{1}}^{*}=\operatorname{Ult}\left(V\left[G_{\alpha}\right],\left(U_{\alpha_{1}}^{\alpha}\right)^{*}\right)$. We want to show that $j_{\alpha_{1}}^{*}\left(g_{\alpha_{0}}^{\alpha_{1}}\right)(\alpha)=\left(U_{\alpha_{0}}^{\alpha}\right)^{*}$. Write $j_{\alpha_{1}}: V \rightarrow M_{\alpha_{1}}=\operatorname{Ult}\left(V, U_{\alpha_{1}}^{\alpha}\right)$. Since $\left|\mathbb{M}_{\alpha}\right|<\alpha$, $j_{\alpha_{1}}$ " $G_{\alpha}$ is generic for $j_{\alpha_{1}}\left(\mathbb{M}_{\alpha}\right)$ over $M_{\alpha_{1}}$, and $j_{\alpha_{1}}^{*}$ is equal to the lift of $j_{\alpha_{1}}$ to $V\left[G_{\alpha}\right] \rightarrow M_{\alpha_{1}}\left[j_{\alpha_{1}} " G_{\alpha}\right]=M_{\alpha_{1}}^{*}$. Therefore $j_{\alpha_{1}}^{*}\left(f_{\alpha_{0}}^{\alpha_{1}}\right)(\alpha)=U_{\alpha_{0}}^{\alpha}$ and so $j_{\alpha_{1}}^{*}\left(g_{\alpha_{0}}^{\alpha_{1}}\right)(\alpha)$ is the ultrafilter generated by $U_{\alpha_{0}}^{\alpha}$ in $M_{\alpha_{1}}^{*}$, which is $\left(U_{\alpha_{0}}^{\alpha}\right)^{*}$.

Now assume that $\alpha$ is a limit point of $A$. For each $i<\beta_{\alpha}$ define $\left(U_{i}^{\alpha}\right)^{*}$ as in the statement of the recursion hypothesis. We prove that the sequence $\left\langle\left(U_{i}^{\alpha}\right)^{*}: i<\beta_{\alpha}\right\rangle$ is $\triangleleft$-increasing. 
Fix $\alpha_{0}<\alpha_{1}<\beta_{\alpha}$ and define $g_{\alpha_{0}}^{\alpha_{1}}$ as follows. The domain of $g_{\alpha_{0}}^{\alpha_{1}}$ is the set of $\xi<\alpha$ such that $\xi$ is a limit point of $A$ and $f_{\alpha_{0}}^{\alpha_{1}}(\xi)$ is a normal ultrafilter in $V$ on $\xi$ which contains $\xi \backslash A$. Note that the domain of $g_{\alpha_{0}}^{\alpha_{1}}$ is in $U_{\alpha_{1}}^{\alpha}$. Let $g_{\alpha_{0}}^{\alpha_{1}}(\xi)$ be equal to $\left(f_{\alpha_{0}}^{\alpha_{1}}(\xi)\right)^{*}$ as defined in the statement of the recursion hypothesis in the model $V\left[G_{\alpha} \uparrow \beta\right]$. Let $\dot{g}_{\alpha_{0}}^{\alpha_{1}}$ be a name such that $\mathbb{M}_{\alpha}$ forces that $\dot{g}_{\alpha_{0}}^{\alpha_{1}}$ satisfies the definition of $g_{\alpha_{0}}^{\alpha_{1}}$ just given.

Write $j_{\alpha_{1}}: V \rightarrow M_{\alpha_{1}}=\operatorname{Ult}\left(V, U_{\alpha_{1}}^{\alpha}\right)$ and $j_{\alpha_{1}}^{*}: V\left[G_{\alpha}\right] \rightarrow M_{\alpha_{1}}^{*}=\operatorname{Ult}\left(V\left[G_{\alpha}\right],\left(U_{\alpha_{1}}^{\alpha}\right)^{*}\right)$. Let $U^{\prime}=j_{\alpha_{1}}^{*}\left(g_{\alpha_{0}}^{\alpha_{1}}\right)(\alpha)$. We want to show that $U^{\prime}=\left(U_{\alpha_{0}}^{\alpha}\right)^{*}$.

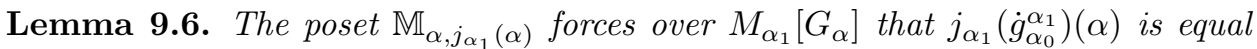
to $\left(U_{\alpha_{0}}^{\alpha}\right)^{*}$.

Proof. By the recursion hypothesis, $M_{\alpha_{1}}\left[G_{\alpha}\right]$ correctly computes $\left(U_{\alpha_{0}}^{\alpha}\right)^{*}$. Since $j_{\alpha_{1}}\left(f_{\alpha_{0}}^{\alpha_{1}}\right)(\alpha)=U_{\alpha_{0}}^{\alpha}$, the lemma follows.

Lemma 9.7. The set of $\xi<\alpha$ such that $g_{\alpha_{0}}^{\alpha_{1}}(\xi)$ is an ultrafilter in $V\left[G_{\alpha}\right]$ is in $\left(U_{\alpha_{1}}^{\alpha}\right)^{*}$.

Proof. Let $\dot{B}$ be a name such that $\mathbb{M}_{\alpha}$ forces that $\dot{B}$ is the set of $\xi<\alpha$ in the domain of $\dot{g}_{\alpha_{0}}^{\alpha_{1}}$ such that $\dot{g}_{\alpha_{0}}^{\alpha_{1}}(\xi)$ is an ultrafilter in $V\left[G_{\alpha}\right]$. To show that $\dot{B}^{G_{\alpha}}$ is in $\left(U_{\alpha_{1}}^{\alpha}\right)^{*}$ it suffices to find $s \leq \leq^{*} 1$ in $\mathbb{M}_{\alpha, j_{\alpha_{1}}(\alpha)}$ which forces over $M_{\alpha_{1}}\left[G_{\alpha}\right]$ that $j_{\alpha_{1}}\left(g_{\alpha_{0}}^{\alpha_{1}}\right)(\alpha)$ is an ultrafilter. Since $j_{\alpha_{1}}\left(g_{\alpha_{0}}^{\alpha_{1}}\right)(\alpha)$ is in ultrafilter in $M_{\alpha_{1}}\left[G_{\alpha}\right]$, it suffices to find $s \leq^{*} 1$ which forces that no new subsets of $\alpha$ are added over $M_{\alpha_{1}}\left[G_{\alpha}\right]$.

Define $s$ as follows. For $\xi$ in $j_{\alpha_{1}}(A) \backslash \alpha$ let $s(\xi)$ be a name for the maximal condition intersected with $\xi \backslash(\alpha+1)$. By Lemma 9.2, s forces that every subset of $\alpha$ is in $M_{\alpha_{1}}\left[G_{\alpha}\right]$.

It follows that $U^{\prime}$ is an ultrafilter in $M_{\alpha_{1}}^{*}$ and hence in $V\left[G_{\alpha}\right]$. So to prove that $U^{\prime}=\left(U_{\alpha_{0}}^{\alpha}\right)^{*}$, it suffices to prove $\left(U_{\alpha_{0}}^{\alpha}\right)^{*} \subseteq U^{\prime}$.

Lemma 9.8. If $X \subseteq \alpha$ in $V\left[G_{\alpha}\right]$, then $X$ is represented in $M_{\alpha_{1}}^{*}$ by the function $\beta \mapsto X \cap \beta$. So $X$ is in $U^{\prime}$ iff the set

$$
\left\{\beta<\alpha: X \cap \beta \in g_{\alpha_{0}}^{\alpha_{1}}(\beta)\right\}
$$

is in $\left(U_{\alpha_{1}}^{\alpha}\right)^{*}$.

The proof is standard. Suppose that $X$ is in $\left(U_{\alpha_{0}}^{\alpha}\right)^{*}$. Let $\dot{X}$ be a name for $X$ such that $\mathbb{M}_{\alpha}$ forces that $\dot{X}$ is a subset of $\alpha$. Let $\dot{Y}$ be a name such that $\mathbb{M}_{\alpha}$ forces

$$
\dot{Y}=\left\{\beta<\alpha: \dot{X} \cap \beta \in \dot{g}_{\alpha_{0}}^{\alpha_{1}}(\beta)\right\} \text {. }
$$

By Lemma 9.8, if $\dot{Y}^{G_{\alpha}}$ is in $\left(U_{\alpha_{1}}^{\alpha}\right)^{*}$, then $X$ is in $U^{\prime}$. By the definition of $\left(U_{\alpha_{1}}^{\alpha}\right)^{*}$, it suffices to prove there is $s$ which directly extends 1 in $\mathbb{M}_{\alpha, j_{\alpha_{1}}(\alpha)}$ such that $s$ forces over $M_{\alpha_{1}}\left[G_{\alpha}\right]$ that $\alpha$ is in $j_{\alpha_{1}}(\dot{Y})$. In other words, $s$ must force

$$
j_{\alpha_{1}}(\dot{X}) \cap \alpha \in j_{\alpha_{1}}\left(\dot{g}_{\alpha_{0}}^{\alpha_{1}}\right)(\alpha)
$$

By Lemma 9.6. it suffices to find $s \leq^{*} 1$ which forces that

$$
j_{\alpha_{1}}(\dot{X}) \cap \alpha \in\left(U_{\alpha_{0}}^{\alpha}\right)^{*} \text {. }
$$

Since $X$ is in $\left(U_{\alpha_{0}}^{\alpha}\right)^{*}$, the following lemma completes the proof.

Lemma 9.9. There is $s \leq^{*} 1$ in $\mathbb{M}_{\alpha, j_{\alpha_{1}}(\alpha)}$ which forces over $M_{\alpha_{1}}\left[G_{\alpha}\right]$ that $X$ is a subset of $j_{\alpha_{1}}(\dot{X}) \cap \alpha$. 
Proof. Fix $\beta$ in $X$. Let $p_{\beta}$ be a condition in $G_{\alpha}$ which forces that $\beta$ is in $\dot{X}$. Write $j_{\alpha_{1}}\left(p_{\beta}\right)=p_{\beta} \widehat{s} s_{\beta}$ where $s_{\beta} \leq^{*} 1$ in $\mathbb{M}_{\alpha, j_{\alpha_{1}}(\alpha)}$. Then $s_{\beta}$ forces over $M_{\alpha_{1}}\left[G_{\alpha}\right]$ that $j_{\alpha_{1}}(\beta)=\beta$ is in $j_{\alpha_{1}}(\dot{X}) \cap \alpha$. So $s_{\beta}$ forces that $\beta$ is in the desired set. By Lemma 9.1 there is $s$ such that $s \leq^{*} s_{\beta}$ for all such $\beta$.

By Proposition $2.1, \mathbb{M}_{\alpha}$ forces that there is a coherent sequence on $\alpha$ as desired. Let $\mathbb{R}_{\alpha}$ be a name for the Radin forcing defined from such a sequence. This completes the construction of the iteration.

\section{REFERENCES}

[1] J. Cummings. Collapsing successors of singulars. Proceedings of the American Mathematical Society, 125:2703-2709, 1997.

[2] M. Gitik. Prikry type forcings. To appear.

[3] T. Jech. Set Theory. Springer, 1997. Second edition.

[4] A. Kanamori. The Higher Infinite. Springer, 1994.

[5] H. Woodin M. Foreman. The generalized continuum hypothesis can fail everywhere. Annals of Mathematics, 133:1-35, 1991.

[6] M. Magidor. How large is the first strongly compact cardinal? Annals of Mathematical Logic, 10(1):33-57, 1976.

[7] W. Mitchell. How weak is a closed unbounded ultrafilter. Logic Colloquium '80.

[8] L. Radin. Adding closed cofinal sequences to large cardinals. Annals of Mathematical Logic, 23:243-262, 1982.

Department of Mathematics, University of California, Berkeley

E-mail address: jkrueger@math.berkeley.edu

URL: http://www. math. berkeley.edu/ jkrueger 